\title{
ON THE RATES OF TYPE Ia SUPERNOVAE IN DWARF AND GIANT HOSTS WITH ROTSE-IIIb
}

\author{
Robert M. Quimby ${ }^{1}$, Fang Yuan ${ }^{2}$, Carl Akerlof $^{3}$, J. Craig Wheeler $^{4}$, And Michael S. Warken ${ }^{5}$ \\ ${ }^{1}$ Kavli IPMU, University of Tokyo, 5-1-5 Kashiwanoha, Kashiwa-shi, Chiba 277-8583, Japan \\ ${ }^{2}$ Research School of Astronomy and Astrophysics, The Australian National University, Weston Creek, ACT 2611, Australia \\ ${ }^{3}$ Physics Department, University of Michigan, Ann Arbor, MI 48109, USA \\ ${ }^{4}$ Department of Astronomy, McDonald Observatory, University of Texas, Austin, TX 78712, USA \\ ${ }^{5}$ Theoretical Division, Mail Stop B227, Los Alamos National Laboratory, Los Alamos, NM 87545, USA \\ Received 2012 March 5; accepted 2012 October 10; published 2012 November 9
}

\begin{abstract}
We present a sample of 23 spectroscopically confirmed Type Ia supernovae ( $\mathrm{SNe}$ Ia) that were discovered in the background of galaxy clusters targeted by ROTSE-IIIb and use up to 18 of these to determine the local $(\bar{z}=0.05)$ volumetric rate. Since our survey is flux limited and thus biased against fainter objects, the pseudo-absolute magnitude distribution (pAMD) of SNe Ia in a given volume is an important concern, especially the relative frequency of high- to low-luminosity SNe Ia. We find that the pAMD derived from the volume-limited Lick Observatory Supernova Search (LOSS) sample is incompatible with the distribution of SNe Ia in a volume-limited $(z<0.12)$ sub-sample of the Sloan Digital Sky Survey II (SDSS-II). The LOSS sample requires far more low-luminosity SNe Ia than the SDSS-II can accommodate. Even though LOSS and SDSS-II have sampled different SNe Ia populations, their volumetric rates are surprisingly similar. Using the same model pAMD adopted in the SDSS-II SNe Ia rate calculation and excluding two high-luminosity SNe Ia from our sample, we derive a rate that is marginally higher than previous low-redshift determinations. With our full sample and the LOSS pAMD, our rate is more than double the canonical value. We also find that 5 of our $18 \mathrm{SNe}$ Ia are hosted by very low luminosity $\left(M_{B}>-16\right)$ galaxies, whereas only 1 out of 79 nearby SDSS-II SNe Ia have such faint hosts. It is possible that previous works have undercounted either low-luminosity SNe Ia, SNe Ia in low-luminosity hosts, or peculiar SNe Ia (sometimes explicitly), and the total SNe Ia rate may be higher than the canonical value.
\end{abstract}

Key word: supernovae: general

Online-only material: color figures

\section{INTRODUCTION}

Calculating the rate of Type Ia supernovae (SNe Ia) and its dependence on redshift illuminates an important contributor to the metal enrichment history of the universe, the production rate of a specific cross section of stellar systems, and, when linked to star formation history, it can reveal the nature and relative fractions of the progenitor systems. Theory and recent empirical evidence argues that the explosions are derived from degenerate, hydrogen depleted stars, namely, white dwarfs (Hoyle \& Fowler 1960; Nugent et al. 2011). The nature of the companion star that must be present to feed mass onto these otherwise stable constructions, however, is more controversial.

It could very well be that there are two or more pathways leading to what we observe as $\mathrm{SNe}$ Ia, including events from single degenerate progenitors (e.g., a white dwarf accreting from a red giant star; Whelan \& Iben 1973) and double degenerate progenitors (i.e., two white dwarfs; Webbink 1984). This is a potential obstacle for the further use of SNe Ia as cosmological probes if the local population used to calibrate the peak magnitude to light curve width relation differs systematically from the distant, cosmologically significant population (i.e., if high-redshift events obey a different relation, cosmology studies may be biased; Domínguez et al. 2001; Mannucci et al. 2006; Sullivan et al. 2006; Howell et al. 2007; Quimby et al. 2007a).

The SNe Ia rate is one tool for resolving this issue. Locally, the quantity of progenitor systems demanded by the SNe Ia rate may be compared to the actual supply available (e.g., Nelemans et al. 2005; Kilic et al. 2012; Badenes \& Maoz 2012). At greater distances, the delay time distribution (DTD), the distribution of $\mathrm{SNe}$ Ia progenitor systems lifespans from birth to explosion, can be compared to the distributions expected form various progenitor models (Yungelson \& Livio 2000). The DTD can be recovered using the SNe Ia rate and star formation histories of a targeted population of hosts (Maoz et al. 2011), or by comparing $\mathrm{SNe}$ Ia over a range of redshifts to the cosmic star formation history (e.g., Mannucci et al. 2006; Graur et al. 2011). The results are consistent with a power-law distribution that favors short delays (for a review, see Maoz \& Mannucci 2012).

Indeed, SNe Ia appear to occur more frequently in latetype galaxies with active star formation than in ellipticals (e.g., Mannucci et al. 2005; Sullivan et al. 2006). Recently, the Lick Observatory Supernova Search (LOSS) reported that the rate of SNe Ia per unit luminosity of the hosts is higher for lowluminosity galaxies than luminous ones ( $\mathrm{Li}$ et al. 2011a). This so-called rate-size relation may be connected to a metallicity effect (Kobayashi \& Nomoto 2009; Kistler et al. 2011), since lower luminosity hosts tend to have lower global metallicities (Tremonti et al. 2004). White dwarfs that form in low-metallicity environments may have a mass distribution that is biased to more massive objects as compared with solar metallicity populations (Umeda et al. 1999), and this in turn may lead to a greater supply of SNe Ia progenitors.

Measurement of the SNe Ia rate was originally performed through searches targeting specific galaxies, and thus the rates derived were in units of the number of SNe Ia found per time per galaxy (Zwicky 1938). This evolved into a rate per galaxy luminosity - $\mathrm{SNuB}$ or $\mathrm{SNuK}$, which give the number of $\mathrm{SNe}$ Ia per $10^{10} L_{\odot}$ (in the $B$ or $K$ bands, respectively), per century. Cappellaro et al. (1999) combined targeted photographic and visual surveys and found an $\mathrm{SNe}$ Ia rate of $\mathrm{SNuB}=0.2 \pm 0.06$ averaged over all galaxy types. LOSS finds roughly compatible 


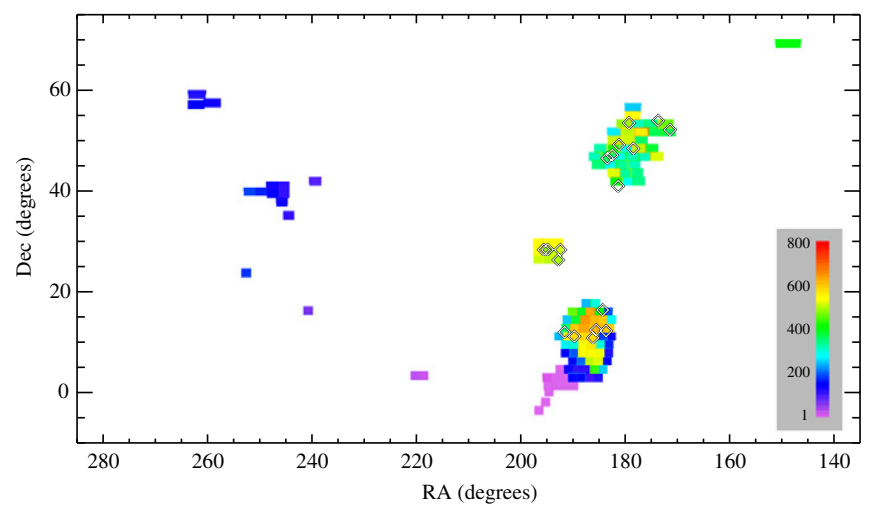

Figure 1. Winter-Spring search fields for the ROTSE-IIIb surveys. The color scale indicates the total number of nights each field was surveyed (i.e., reference epochs are not included). The diamonds mark the locations of background $\mathrm{SNe}$ Ia.

(A color version of this figure is available in the online journal.)

SNe Ia SNuB rates in specific host types if they do not include the rate-size relation ( $\mathrm{Li}$ et al. 2011a). They also use an adopted $K$-band galaxy luminosity function (Kochanek et al. 2001) to convert their measurements into volumetric rates.

Methods to discover SNe Ia without preference for host galaxies (e.g., Hamuy et al. 1993; Perlmutter et al. 1997) have been widely adopted as the availability of wide-field cameras has increased (e.g., SNLS, ESSENCE, SDSS-II, PTF, PanSTARRS, and SkyMapper). SNe Ia rates from such surveys are most

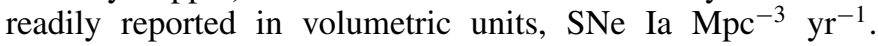
Included in this survey category is the Sloan Digital Sky Survey II (SDSS-II), which was designed to bridge the gap between the local SNe Ia and the high-redshift events used as cosmological probes (Frieman et al. 2008). Over a three year period, the SDSS-II spectroscopically confirmed several hundred SNe Ia in the range $0 \lesssim z \lesssim 0.4$. Dilday et al. (2010) use a sub-sample of (normal) SNe Ia discoveries to derive the $z<0.3$ rate, which is seen to increase roughly as a power law with redshift, $\mathcal{R} \propto(1+z)^{2}$. SNe Ia rate studies have been conducted at even higher redshifts, including work done with the Supernova Legacy Survey (SNLS; Neill et al. 2006; Perrett et al. 2012), Hubble Space Telescope (Dahlen et al. 2008), and Subaru (Graur et al. 2011). The measured SNe Ia rates increase to $z \sim 1$, and then level off. For a recent compilation of volumetric SNe Ia rates from various surveys, see Graur et al. (2011).

In this paper, we determine the volumetric SNe Ia rate and contributions to this from dwarf and giant host galaxies based on discoveries from the ROTSE-IIIb telescope. Section 2 discusses how our sample was selected. We discuss our unfiltered magnitude system and how this may relate to $\mathrm{SNe}$ Ia pseudoabsolute magnitude distributions (pAMDs) constructed from filtered observations in Section 3. We also discuss the pAMDs derived from the LOSS and SDSS-II in this section. For the later, we determine an empirical pAMD based on a volumelimited sub-sample of the SDSS-II after discussing the pAMD model that was actually used in the SDSS-II SNe Ia rate calculation (and which we adopt for comparison to the SDSS-II). In Section 4, we study our ability to detect point sources of various brightness as a function of seeing and limiting magnitudes, and we use this in Section 5 to determine our overall survey efficiency - the probability of discovering SNe Ia drawn from a given population-as a function of distance. Next, we measure multi-band photometry for the host galaxies of our supernovae and calculate rest-frame absolute magnitudes in

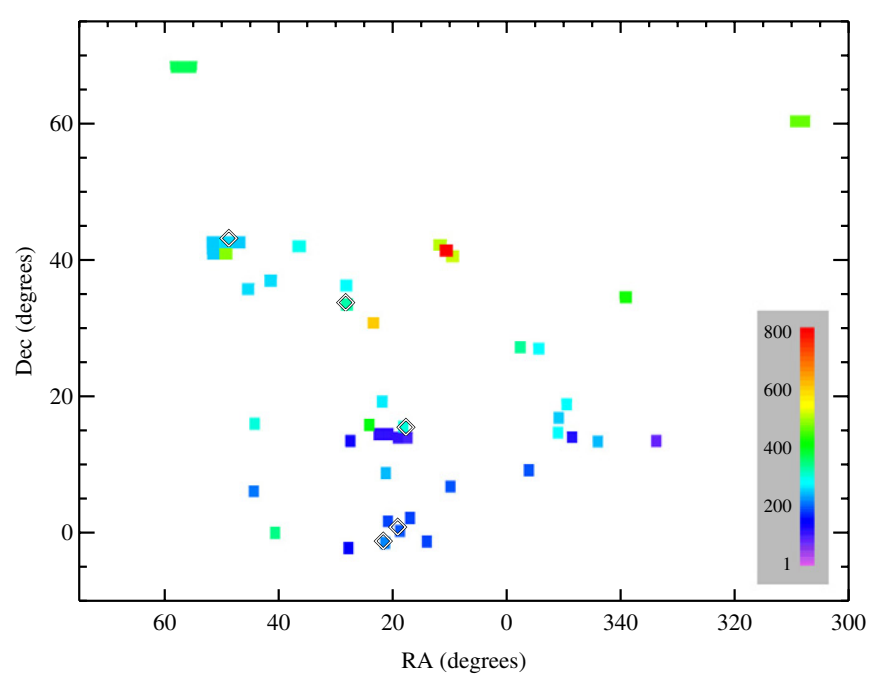

Figure 2. Same as Figure 1, but for the Summer-Fall fields. (A color version of this figure is available in the online journal.)

Section 6. SNe Ia rates are calculated in Section 7, and final conclusions are offered in Section 8. Throughout this paper, we assume a flat, $H_{0}=71 \mathrm{~km} \mathrm{~s}^{-1} \mathrm{Mpc}^{-1}$ and $\Omega_{m}=0.27$ cosmology.

\section{SAMPLE}

Our sample is drawn from the background population of supernovae discovered by ROTSE-IIIb in the course of the Texas Supernova Search (TSS; Quimby 2006) and the ROTSE Supernova Verification Project (RSVP; Yuan et al. 2007a). Details of the full ROTSE-IIIb supernova sample will be presented in a forthcoming paper. Here, we summarize the key characteristics of the sample selection process.

The TSS began survey operations in November of 2004 and was succeeded by RSVP in early 2008. Our survey instrument, ROTSE-IIIb, has a $0.45 \mathrm{~m}$ aperture and a $1.85 \times 1.85 \mathrm{deg}$ field of view. The search continues to operate and has been expanded to the other ROTSE-III telescopes in Australia, Namibia, and Turkey, but for this work we consider only discoveries made by ROTSE-IIIb in Texas prior to 2009 February.

We typically targeted galaxy clusters such as Virgo, Ursa Major, Coma, and Abell clusters for our supernova search, but in a few exceptional cases we chose to target specific galaxies (e.g., M31). In the present work, we remove this potential source of bias by selecting only the subset of SNe Ia found in the background of each field $\left(z>z_{f}+4000 \mathrm{~km} \mathrm{~s}^{-1}\right.$, where $z_{f}$ is the redshift of the object targeted in a given field). During the search period, we detected 76 supernovae in total, and of these 46 were classified as SNe Ia. After removing foreground and cluster supernovae, we are left with the 23 SNe Ia background events listed in Table 1. Figures 1 and 2 show our sky coverage with the locations of the discoveries marked. Fields with the highest concentrations of nearby galaxies were typically observed every night as weather and season allowed. Over the first few years, we observed the less rich fields on alternating nights. Additional time was allocated for the survey in the RSVP era, and fields were added at that time.

For both TSS and RSVP, we employed image subtraction techniques to reveal time-variable events. The TSS employed a modified version of the Perlmutter et al. (1999) search code, and the RSVP used the process described in Yuan \& Akerlof (2008). For our survey, we typically observe each field multiple 
Table 1

Background SN Ia Discovered with ROTSE-IIIb by 2009 February 1

\begin{tabular}{|c|c|c|c|c|c|c|c|c|c|c|}
\hline IAU Name & Disc. Date & R.A. & Decl. & $z$ & $\begin{array}{l}\text { Disc. } \\
\text { Mag }\end{array}$ & $N_{\text {det }}$ & $\begin{array}{l}\text { Abs. } \\
\text { Mag }\end{array}$ & $\begin{array}{l}\text { Used? } \\
\text { LOSS }\end{array}$ & $\begin{array}{c}\text { Used? } \\
\text { SDSS-II }\end{array}$ & Note \\
\hline $2004 \mathrm{gu}$ & 2004 Dec 13 & $12: 46: 24.7$ & $+11: 56: 56$ & 0.05 & 17.4 & 6 & -19.4 & $\mathrm{y}$ & $\mathrm{y}$ & \\
\hline $2005 \mathrm{bg}$ & 2005 Mar 28 & $12: 17: 17.1$ & $+16: 22: 17$ & 0.02 & 17.1 & 10 & -19.0 & $\mathrm{y}$ & $\mathrm{y}$ & \\
\hline $2005 \mathrm{ck}$ & 2005 Jun 5 & $13: 02: 18.7$ & $+28: 20: 44$ & 0.09 & 18.6 & 2 & -19.9 & $\mathrm{n}$ & $\mathrm{n}$ & a \\
\hline $2005 \mathrm{cr}$ & 2005 Jun 24 & $12: 22: 17.1$ & $+12: 23: 49$ & 0.02 & 16.1 & 10 & -19.1 & $\mathrm{y}$ & $\mathrm{y}$ & $\mathrm{c}$ \\
\hline $2005 \mathrm{hj}$ & 2005 Oct 30 & $01: 26: 48.3$ & $-01: 14: 17$ & 0.06 & 17.6 & 17 & -19.7 & $\mathrm{y}$ & $\mathrm{y}$ & $\mathrm{b}$ \\
\hline $2005 \mathrm{ir}$ & 2005 Nov 3 & $01: 16: 43.7$ & $+00: 47: 40$ & 0.08 & 18.5 & 6 & -19.4 & $\mathrm{y}$ & $\mathrm{y}$ & $\mathrm{b}$ \\
\hline 2006an & 2006 Feb 21 & $12: 14: 38.7$ & $+12: 13: 47$ & 0.06 & 18.0 & 2 & -19.4 & $\mathrm{n}$ & $\mathrm{n}$ & \\
\hline $2006 \mathrm{cj}$ & 2006 May 17 & $12: 59: 24.5$ & $+28: 20: 51$ & 0.07 & 17.9 & 11 & -19.5 & $\mathrm{y}$ & $\mathrm{y}$ & $\mathrm{c}$ \\
\hline $2006 \mathrm{ct}$ & 2006 May 25 & $12: 09: 56.8$ & $+47: 05: 45$ & 0.03 & 17.5 & 11 & -18.6 & $\mathrm{y}$ & $\mathrm{y}$ & \\
\hline 2007if & 2007 Aug 16 & 01:10:51.4 & $+15: 27: 40$ & 0.07 & 19.5 & 12 & -20.6 & $\mathrm{y}$ & $\mathrm{n}$ & d \\
\hline $2007 \mathrm{kh}$ & 2007 Sep 7 & $03: 15: 12.1$ & $+43: 10: 13$ & 0.05 & 18.9 & 10 & -19.3 & $\mathrm{y}$ & $\mathrm{y}$ & \\
\hline 2007op & 2007 Nov 4 & $01: 53: 12.4$ & $+33: 44: 34$ & 0.09 & 18.5 & 6 & -19.1 & $\mathrm{y}$ & $\mathrm{y}$ & \\
\hline $2007 q c$ & 2007 Oct 27 & $11: 57: 04.7$ & $+53: 29: 55$ & 0.04 & 16.8 & 11 & -19.1 & $\mathrm{y}$ & $\mathrm{y}$ & \\
\hline $2007 \mathrm{sp}$ & 2007 Nov 14 & $12: 04: 42.3$ & $+49: 11: 09$ & 0.02 & 16.8 & 7 & -18.2 & $\mathrm{y}$ & $\mathrm{y}$ & $\mathrm{e}$ \\
\hline $2007 \mathrm{sw}$ & 2007 Dec 29 & $12: 13: 36.9$ & $+46: 29: 36$ & 0.03 & 16.0 & 2 & -18.8 & $\mathrm{n}$ & $\mathrm{n}$ & \\
\hline $2008 \mathrm{E}$ & 2008 Jan 4 & $11: 25: 37.0$ & $+52: 08: 26$ & 0.03 & 18.2 & 18 & -18.7 & $\mathrm{y}$ & $\mathrm{y}$ & \\
\hline $2008 \mathrm{ab}$ & 2008 Jan 30 & $11: 34: 45.9$ & $+53: 57: 51$ & 0.07 & 18.2 & 8 & -19.7 & $\mathrm{y}$ & $\mathrm{n}$ & \\
\hline $2008 \mathrm{ac}$ & 2008 Jan 30 & $11: 53: 45.2$ & $+48: 25: 22$ & 0.05 & 17.6 & 15 & -19.2 & $\mathrm{y}$ & $\mathrm{y}$ & \\
\hline 2008ad & 2008 Jan 30 & $12: 49: 37.2$ & $+28: 19: 47$ & 0.05 & 18.0 & 2 & -19.1 & $\mathrm{n}$ & $\mathrm{n}$ & \\
\hline 2008ar & 2008 Feb 27 & $12: 24: 37.9$ & $+10: 50: 17$ & 0.03 & 16.9 & 17 & -18.8 & $\mathrm{y}$ & $\mathrm{y}$ & \\
\hline $2008 \mathrm{bg}$ & 2008 Mar 12 & $12: 51: 11.9$ & $+26: 17: 40$ & 0.06 & 18.7 & 9 & -19.3 & $\mathrm{y}$ & $\mathrm{y}$ & c \\
\hline $2008 b y$ & 2008 Apr 19 & $12: 05: 21.0$ & $+40: 56: 46$ & 0.05 & 17.2 & 2 & -19.8 & $\mathrm{n}$ & $\mathrm{n}$ & \\
\hline $2008 \mathrm{bz}$ & 2008 Apr 22 & $12: 38: 57.7$ & $+11: 07: 46$ & 0.06 & 17.7 & 6 & -19.2 & $\mathrm{y}$ & $\mathrm{y}$ & \\
\hline
\end{tabular}

Notes.

a Also reported by LOSS (Pugh et al. 2005).

b Also detected by SDSS-II and used in their volumetric rate calculations (Dilday et al. 2008, 2010).

c Spectroscopically confirmed by the CfA (Modjaz et al. 2005; Colesanti et al. 2006; Yuan et al. 2008b).

d Also reported by the Nearby SN Factory (Yuan et al. 2007b; Scalzo et al. 2010).

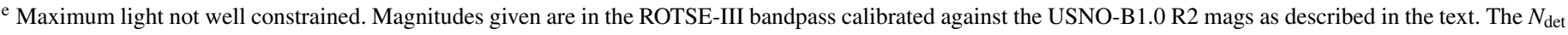

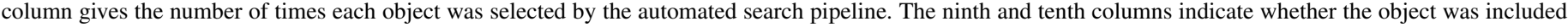
in the rate calculations using the LOSS pAMD or the SDSS-II model pAMD, respectively.

times each night. From these we create three co-added frames representing the average of the first half of the nights data (NEW1), the average of the second half (NEW2), and the average for the whole night (NEW). We use filtering to remove particle events and other artifacts during the co-addition. We then subtract the properly convolved reference template from each of these to generate subtracted frames (SUB1, SUB2, and SUB). To remove contamination from solar system bodies and remaining artifacts, we require a candidate to be detected $(\gtrsim 2.5 \sigma)$ at consistent celestial coordinates on each of SUB1 and SUB2. The candidate is finally required to be detected at the $5 \sigma$ confidence level on the combined subtraction, SUB. The typical FWHM for the survey was 3.2 pixels (about $10^{\prime \prime}$ ) and the $3 \sigma$ limiting magnitude for the nightly co-adds was typically around $18.3 \mathrm{mag}$.

We further vet potential SN candidates against catalogs of known time-variable phenomena. ${ }^{6}$ Most of our fields were observed by the SDSS as well, so we were able to remove contamination from uncataloged variable stars by checking for matching "host" stars in the SDSS data, which is complete to $\sim 4$ mag below our survey depth. Remaining sources were vetted by human scanners. All sources judged as possible transients were spectroscopically classified by us or others in the community. This is a key distinguishing feature of our survey; other wide-field transient searches generate more candidate supernovae than can be classified with the spectroscopic resources available, so a

6 For example: http://scully.cfa.harvard.edu/cgi-bin/checkmp.cgi, http://simbad.u-strasbg.fr/simbad/sim-fid, and http://nedwww.ipac.caltech.edu/ filtering process such as light curve fitting is typically employed to select only a subset for final spectroscopic confirmation.

Table 1 also notes the number of times that each discovery was recovered by the automated search pipeline $\left(N_{\mathrm{det}}\right)$. A final analysis of the data adds low significance (i.e., $<5 \sigma$ ) detections that are not passed by the automated selection cuts, and we determine the peak magnitudes for each supernova in our sample by fitting light curve templates to these final data sets. We use $R$-band light curve templates for $\mathrm{SNe}$ Ia from LOSS, which were constructed by interpolating between the faint/fast SN 1999by and the bright/slow SN 1991T (Li et al. 2011b). See Section 3 for a discussion of our unfiltered spectral response and a comparison to the $R$ band. We use mpfit.pro in IDL to determine the best-fitting template shape, and subtract off the Galactic extinction in the $R$ band (Schlegel et al. 1998). We employ the distance modulus (based on independent distance indicators when available through $\mathrm{NED}^{7}$ or otherwise based on the redshift in a flat, $H_{0}=71 \mathrm{~km} \mathrm{~s}^{-1} \mathrm{Mpc}^{-1}$ and $\Omega_{m}=0.27$ cosmology) to determine the peak absolute magnitudes shown in Table 1. SN 2004gu was first observed near peak, which adds some uncertainty to the date and magnitude of maximum light; however, spectroscopic phase information is consistent with the derived date of maximum light, and thus we expect the peak magnitude estimate to be accurate. According to its spectroscopic age, another event, SN2007sp, was first detected about two months after maximum light, so the light curve fit likely underestimates the peak.

\footnotetext{
7 http://ned.ipac.caltech.edu/
} 


\subsection{Sample Notes}

Although our sample is small, it contains several SNe Ia worthy of special note.

1. SN 2004gu is a significant outlier on the Hubble diagram, and other authors have grouped it with peculiar events such as SN 2006gz and SN 1999ac, which may or may not be linked to super-Chandrashekar explosions (Contreras et al. 2010; Silverman et al. 2011).

2. SN 2006ct showed the characteristic features of an SN Ia, but the blueshift of the line minima near maximum light was only $\sim 6000 \mathrm{~km} \mathrm{~s}^{-1}$ (Quimby et al. 2006). The spectra show some similarities to the highly peculiar SN 2002es (Ganeshalingam et al. 2012), which was also hosted by an early-type galaxy, but the photometric decline of SN 2006ct (Quimby 2006) is slower and more reminiscent of SN 2002cx (Li et al. 2003) and SN 2005hk (Phillips et al. 2007).

3. SN 2007if is the most luminous SN Ia known, and it may be the result of a super-Chandrashekar mass explosion (Scalzo et al. 2010; Yuan et al. 2010).

4. SN 2007qc was hosted by an extremely low luminosity dwarf galaxy (see Section 6). At $M_{B} \sim-11$, the host is among the faintest detected for any SNe Ia.

5. SN 2008ar showed spectroscopic evidence for highvelocity material and unusually strong absorption in the Ca II IR triplet (Yuan et al. 2008a), which is reminiscent of SN 2007le (Simon et al. 2009).

In addition, at least seven of our SNe Ia (2004gu, 2005ck, 2005hj, 2006cj, 2007if, 2008ab, 2008by, and possibly 2005bg) show either unusually high luminosities or are spectroscopically classified as SN 1991T/1999aa-like. This appears to be a high fraction even for a magnitude-limited survey when compared with the LOSS census of SNe Ia in targeted galaxies ( $\mathrm{Li}$ et al. 2011b).

\section{SN Ia PSEUDO-ABSOLUTE MAGNITUDE DISTRIBUTIONS}

Our survey is flux limited, and as a result we have a bias against selecting low-luminosity $\mathrm{SNe}$ Ia. This bias is exacerbated by our removal of discoveries made at or below the redshift of the targeted galaxy clusters. To determine the event rate for the $\mathrm{SNe}$ Ia population as a whole, we must therefore correct the number of events in our observed sample for the fraction we are likely to have missed due to, among other concerns, their lower luminosities (see Section 5). This requires knowledge of the intrinsic luminosity function for all SNe Ia in our search volume combined with the host galaxy absorption distribution.

In this section, we consider the pAMD for SNe Ia, which is the distribution one would obtain by correcting the peak observed magnitudes of a complete sample of SNe Ia for distance and Galactic extinction but not for host galaxy extinction. This distribution can directly be used to test the selection efficiency of a flux-limited survey (see Section 5). First, we consider in Section 3.1 how the available SNe Ia luminosity functions derived (mostly) through filtered observations may compare to our unfiltered survey data. We then discuss the pAMD compiled by LOSS from their targeted, but volume-limited sample (Li et al. 2011b) in Section 3.2, and we derive an empirical pAMD from a volume-limited sub-sample of the SDSS-II survey in Section 3.3. We find that the LOSS and SDSS-II pAMDs are not consistent, which is discussed in Section 3.4.

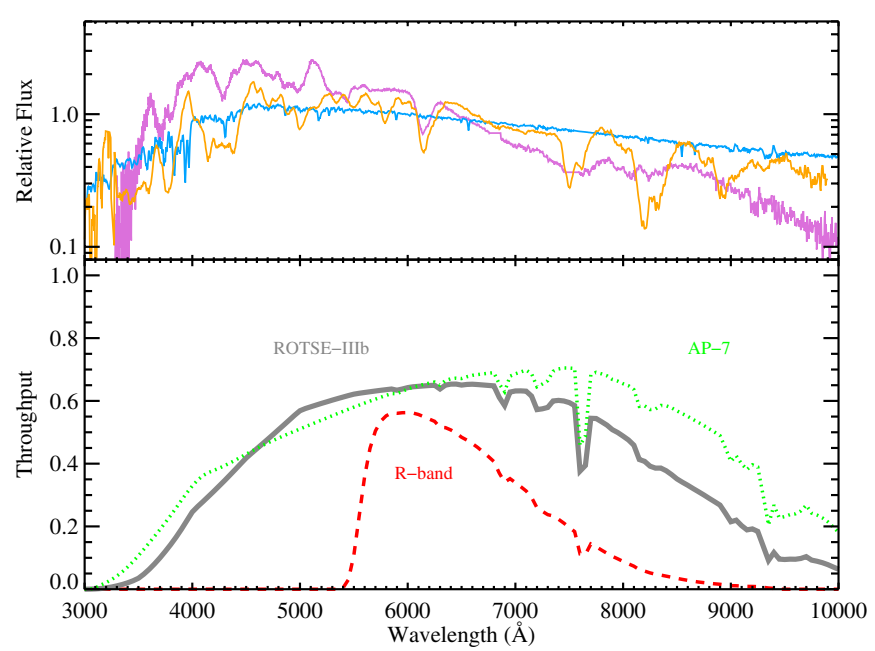

Figure 3. Determination of the magnitude offset between filtered and unfiltered measurements. The top panel shows the spectral energy distributions of a typical field standard (blue) compared to a high-luminosity SN Ia (purple) and a lowluminosity SN Ia (orange). These can be convolved with the ROTSE-IIIb (gray) and $R$-band (dashed red) response curves in the lower panel to determine the magnitude offset. The dotted green curve is the typical response for an Apogee 7 camera, which is used by KAIT for the LOSS. See the text for details.

(A color version of this figure is available in the online journal.)

\subsection{Comparison of $R$-band and ROTSE-IIIb Unfiltered Magnitudes}

We calibrate our instrumental magnitudes against the USNOB1.0 R2 system since some of our search fields are outside of the SDSS footprint. For fields covered by both the SDSS and USNO-B1.0, we compared the USNO-B1.0 photometry to SDSS photometry converted ${ }^{8}$ to the $R$ band using $R=$ $r-0.2936(r-i)-0.1439$, and we find the two systems agree to about $0.01 \mathrm{mag}$ on average and have a field to field dispersion of about 0.14 mag. Thus, our unfiltered ROTSE-IIIb magnitudes should be, on average, similar to the $R$ band, but objects with spectral energy distributions that differ significantly from the field stars employed in the calibration may be offset from their true $R$-band values.

We estimate the potential offset between our unfiltered magnitudes and the true $R$-band system for SNe Ia as follows. We adopt the spectral energy distribution of a G5V star (Pickles 1998) as representative of a typical field standard used in calibrating the photometric zero point of our unfiltered imaging data. We scale this template to zero magnitude in the $R$ band, and then measure the synthetic flux using the approximate transmission function of ROTSE-IIIb (Quimby et al. 2007b), which is plotted in Figure 3. We next scale a template ${ }^{9}$ spectrum for a Branch-normal SN Ia at maximum light to zero magnitude in the $R$ band and measure its flux in the ROTSE-IIIb bandpass. The ratio of this and the scaled field star flux (also measured in the ROTSE-IIIb bandpass) gives the expected departure of the ROTSE-IIIb magnitudes from the true $R$-band system. We measure this offset in the range $0.02<z<0.09$ by redshifting the SN Ia template, and find that our unfiltered magnitudes may be $0.05-0.10 \mathrm{mag}$ brighter than the true $R$-band system. We repeat this process for a high-luminosity SN Ia (SN 1999aa; Garavini et al. 2004), and a low-luminosity event (SN 1991bg; Filippenko et al. 1992). We find that an SN Ia with the same

\footnotetext{
8 http://www.sdss.org/dr7/algorithms/sdssUBVRITransform.html \#Lupton2005

9 http://supernova.lbl.gov/nugent/nugent_templates.html
} 
$R$-band brightness as a field standard will generate a magnitude in the unfiltered system (calibrated against the $R$ band) that is within $\sim 0.1$ mag of its true $R$-band magnitude.

While this shows that our observed magnitudes should be similar to the true $R$-band system, a more pertinent concern is how the derived absolute magnitudes will compare. In particular, we have calculated absolute magnitudes from our unfiltered data by simply subtracting off the distance moduli and Galactic ( $R$ band) extinction terms; we have not included a " $k$-correction" term to account for the changing rest-frame bandpass of our unfiltered system over the modest redshift distribution of our sample. Below, we compare the pseudoabsolute magnitudes derived from our data to rest frame ( $k$-corrected) $R$-band magnitudes calculated for the same sources based on filtered photometry to check for an offset.

The CfA3 sample includes MLCS2k2 (Jha et al. 2007) fits for four of the supernovae in our sample (Hicken et al. 2009), including two that were also in the SDSS-II sample (Kessler et al. 2009). We calculate the pseudo-absolute magnitudes from these fits using the $\Delta$ parameters and adding back in the host extinction term. For this comparison, we calibrate the ROTSE-IIIb magnitudes against the SDSS field stars (converted to the $R$ band as described above) to remove the USNO-B1.0 versus SDSS-II offset, which we find to be biased in this small overlapping sample.

Our pseudo-absolute magnitude estimate for SN 2005hj is about 0.1 mag brighter than the fits from the CfA3 and the SDSS-II. For SN 2005ir, the SDSS-II and CfA3 differ from each other by about $0.1 \mathrm{mag}$, which is comparable to the combined measurement error. Our absolute magnitude for SN 2005ir is about 0.02 mag brighter than the SDSS-II value and 0.09 mag fainter than the CfA3 value. Our measurements of SN 2006an and SN 2006cj are 0.03 mag brighter and 0.16 mag fainter than the CfA3 peak magnitudes, respectively.

Additionally, the Nearby Supernova Factory has reported a peak $V$-band magnitude and $V-R$ color for SN 2007if that implies a peak absolute $R$-band maximum of about -20.4 mag. Calibrating against the SDSS $r$-band AB magnitudes, Yuan et al. (2010) found the ROTSE-III observations peaked at $M_{r}=-20.4$. Field stars will typically "brighten" by about 0.22 mag when converting from the SDSS $r$-band system to Cousins $R$-band (Vega) magnitudes, which shifts the target photometry by the same amount. Our estimate for the peak of SN 2007if is approximately 0.15 mag brighter than the Nearby Supernova Factory value.

To summarize of the seven pseudo-absolute magnitude measurements available in the literature based on ( $k$-corrected) filtered photometry, our ROTSE-IIIb estimates are $\sim 0.1$ mag brighter in three cases, $\sim 0.1$ mag fainter in two cases, and roughly the same in the remaining two cases. On average, our values are 0.02 mag brighter. We conclude that there is no significant systematic offset between our unfiltered, pseudo-absolute magnitude estimates and the (mostly) $R$-band system that defines the luminosity functions considered below (Sections 3.2 and 3.3), and these should agree to about $\sim 0.1$ mag for individual events. We address the impact the possible systematic error in our magnitude system with respect to the LOSS and SDSS-II pAMDs will have on our rates in Section 8.

\subsection{The LOSS Pseudo-absolute Magnitude Distribution for SNe Ia}

The LOSS has recently published a distribution of pseudoabsolute magnitudes for $\mathrm{SNe}$ Ia from their volume-limited search (Li et al. 2011b). Like ROTSE-IIIb, the LOSS survey engine (the Katzman Automatic Imaging Telescope, or KAIT) runs with an unfiltered CCD imager, but follow-up observations are often obtained through standard broadband filters. The SNe Ia luminosity function reported by Li et al. (2011b) is primarily constructed from $R$-band data, which the authors describe as the best match to their unfiltered survey data, and also from unfiltered observations as well. In the lower panel of Figure 3, we show the response curve for the Cousins $R$ band compared to the typical unfiltered response of an Apogee 7 camera, which is used by LOSS.

It is important to note that the LOSS SNe Ia luminosity function was drawn from a sample that was highly biased with respect to the host galaxy properties. In particular, the LOSS mainly targeted high-luminosity galaxies. It has been demonstrated that certain SNe Ia sub-types prefer certain host galaxies (Sullivan et al. 2006), so the luminosity distribution of the LOSS sample may be biased with respect to the larger population from which our sample is drawn.

An obvious difference between the LOSS and ROTSE-IIIb SN Ia samples is the presence of high-luminosity $\left(M_{R}<-19.6\right)$ events in the latter. Although we could follow previous works and simply discard our most luminous SNe Ia, we instead chose to calculate an inclusive rate. We must, therefore, augment the LOSS luminosity function to account for such events or else our effective survey volume will be underestimated. Lacking detailed demographics for this population, we simply assume that the population of high-luminosity SNe Ia represents only about $1 \%$ of SNe Ia in a given volume; if it were much more these would be detected more frequently, and if it were much less we could not expect to find any in our small sample (see Section 5). We assume that SN 2007if was a particularly luminous example of this population (as noted above, it is the most luminous SN Ia known) and choose a half Gaussian with a peak at $M_{R}=-19.6$ and $\sigma=0.4 \mathrm{mag}$ to stand in for the unknown luminosity distribution. We will use this augmented version of the LOSS pAMD to calculate the SNe Ia rate in Section 7.

\subsection{The SDSS-II Pseudo-absolute Magnitude Distribution for SNe Ia}

For our rate calculations in Section 7, we will also use the same bimodal-Gaussian luminosity function attenuated by a host galaxy absorption distribution that was assumed by Dilday et al. (2010) in deriving the SDSS-II rate. The model is a revised version ${ }^{10}$ of the distribution assumed by Dilday et al. (2008) that takes into account the findings of Kessler et al. (2009). The difference makes for only a negligible change in our rate measurement. The intrinsic SNe Ia luminosity function is defined in terms of the MLCS2k2 light curve shape/luminosity parameter, $\Delta$. The distribution of $\Delta$ is defined as a Gaussian with $\sigma=0.19$ for $\Delta<-0.2$ and $\sigma=0.40$ for $\Delta>-0.2$, and it is truncated to lie within the range $(-0.4<\Delta<1.8)$. The final, pAMD is achieved by adding host galaxy absorption drawn from a distribution with $P\left(A_{R}\right) \propto e^{-A_{R} / 0.28}$. With our choice of $H_{0}$, peak pseudo-absolute magnitudes are then defined as (cf. Jha et al. 2007)

$$
M_{R}=-19.313+0.579 \Delta+0.254 \Delta^{2}+A_{R} .
$$

When applying the SDSS-II pAMD model to our ROTSE-IIIb discoveries, it is important to note that the SDSS-II rate is based on a sub-sample of SNe Ia, and to be consistent with

\footnotetext{
10 B. Dilday 2012, private communication.
} 
the SDSS-II pAMD model we must also remove objects from our sample. In particular, the SDSS-II includes only the fraction of the population that is well fit by $\operatorname{MLCS} 2 \mathrm{k} 2\left(\mathcal{P}_{\text {fit }}>0.001\right)$ with a light curve width parameter $\Delta>-0.4$. Such a cut excludes high-luminosity SNe Ia, and in particular, SN 1999aalike events (Kessler et al. 2009). We note that this is a common choice. The SNLS, for example, has presented a volumetric rate for $\mathrm{SNe}$ Ia at $\bar{z} \sim 0.45$, but the spectroscopically confirmed Type Ia SN 2003fg (SNLS-03D3bb) is not included (Neill et al. 2006; Howell et al. 2006). Indeed, the selection cuts that are applied in selecting candidates for spectroscopic follow-up may also pose a bias against high-luminosity $\mathrm{SNe}$ Ia. We therefore remove events with peak luminosities brighter that $M_{R}<-19.7$ when using the model pAMD from the SDSS-II.

We next look at the actual distribution of pseudo-absolute magnitudes from a volume-limited sub-sample of the first year SDSS-II SNe Ia discoveries (Frieman et al. 2008; Sako et al. 2008), which can be compared to the assumed model as well as the LOSS pAMD. According to Dilday et al. (2008), the SDSS-II could have detected even a sub-luminous (SN 1991bglike) SN Ia out to a redshift of $z \sim 0.12$, so we assume the survey to be volume limited out to this limit. There were 29 spectroscopically confirmed (or probable) SNe Ia from the first year of the SDSS-II search within this redshift limit. In addition, Dilday et al. (2008) lists one probable SN Ia (SDSS-II SN09266; based on photometric constraints) with a host redshift of $z=0.0361$. This source is not included in the sample of Dilday et al. (2010) due to an additional cut on the photometric screening. A second photometrically probable source, SDSS-II SN09739, with a spectroscopic redshift for its host, and a third, photometrically probable SN Ia (SDSS-II SN11092) are also given for a total of $32 \mathrm{SNe}$ Ia in the volume-limited sample.

We now derive an empirical SNe Ia pAMD from this volumelimited sub-sample of the first year SDSS-II. We adopt the temporal selection cuts of Dilday et al. (2008) and Kessler et al. (2009) to remove events with poorly constrained maxima, but unlike these samples, ours includes peculiar events. For the bulk of the sample, we derive the peak absolute $R$-band magnitudes from the MLCS2k2 (Jha et al. 2007) fit parameters given in Kessler et al. (2009). We add the host extinction estimate back in to retain the pseudo-absolute distribution required for rate studies. Two peculiar SNe Ia, SN 2005hk and SN 2005gj, were cut from the Kessler et al. (2009) sample; it is still a matter of debate whether these events truly belong to the Type Ia class (e.g., Valenti et al. 2009; Foley et al. 2010; Maund et al. 2010; Aldering et al. 2006; Trundle et al. 2008). We include them here for completeness. For SN 2005hk, we take the LOSS measurement for the $R$-band absolute magnitude (Li et al. 2011b), and for SN 2005gj we take the peak absolute $R$-band magnitude from Prieto et al. (2007) and convert this to $R$ band. We similarly use the SDSS-II photometric measurements of Holtzman et al. (2008) to estimate peak absolute $R$-band magnitudes for two spectroscopically probable SNe Ia: SN 2005je and SDSS-II SN06968. Finally, we choose to include an estimated peak magnitude for SDSS-II SN09266. There is no published light curve or peak magnitude for this event, so we assign it an ad hoc pseudo-absolute magnitude of $M_{R}=-16$ mag based on the heavy extinction reported ( $A_{V} \sim 4$; Dilday et al. 2008).

We thus have peak magnitudes for 22 of the first year SDSS-II SNe Ia; eight of the remaining events have poorly constrained maxima. The portions of the light curves sampled by the SDSS-II, however, imply a similar distribution of peak
Table 2

SDSS-II First Year Volume-limited Sample

\begin{tabular}{|c|c|c|c|}
\hline SDSS ID & IAU Name & $M_{R}$ & Note \\
\hline 722 & 2005 ed & $\cdots$ & \\
\hline 739 & 2005 ef & $\cdots$ & \\
\hline 774 & $2005 \mathrm{ei}$ & $\ldots$ & \\
\hline 1241 & $2005 \mathrm{ff}$ & -18.97 & \\
\hline 1371 & $2005 \mathrm{fh}$ & -19.39 & \\
\hline 2102 & $2005 \mathrm{fn}$ & $\cdots$ & \\
\hline 2561 & $2005 \mathrm{fv}$ & -19.03 & \\
\hline 3256 & $2005 \mathrm{hn}$ & -19.11 & \\
\hline 3592 & $2005 \mathrm{gb}$ & -19.30 & \\
\hline 3901 & 2005ho & -19.33 & \\
\hline 4524 & $2005 \mathrm{gj}$ & -20.47 & $\mathrm{a}$ \\
\hline 5395 & $2005 \mathrm{hr}$ & -19.36 & \\
\hline 5549 & $2005 h x$ & -19.23 & \\
\hline 5944 & 2005hc & -19.41 & \\
\hline 6057 & 2005if & -18.95 & \\
\hline 6295 & $2005 \mathrm{js}$ & -17.72 & \\
\hline 6558 & 2005hj & -19.36 & \\
\hline 6773 & 2005iu & -19.28 & \\
\hline 6962 & $2005 \mathrm{je}$ & -19.28 & $\mathrm{~b}$ \\
\hline 6968 & $\cdots$ & -19.06 & b \\
\hline 7147 & 2005jh & -18.96 & \\
\hline 7876 & 2005ir & -19.29 & \\
\hline 8151 & $2005 \mathrm{hk}$ & -18.36 & $\mathrm{c}$ \\
\hline 8719 & $2005 \mathrm{kp}$ & -19.30 & \\
\hline 9266 & $\cdots$ & -16.00 & d \\
\hline 9739 & $\cdots$ & $\cdots$ & \\
\hline 10028 & $2005 \mathrm{kt}$ & -19.06 & \\
\hline 10096 & 2005lj & $\cdots$ & \\
\hline 10434 & $2005 \mathrm{lk}$ & $\cdots$ & \\
\hline 10805 & $\ldots$ & $\cdots$ & \\
\hline 11067 & $2005 \mathrm{ml}$ & $\cdots$ & \\
\hline 11092 & $\cdots$ & $\cdots$ & \\
\hline
\end{tabular}

Notes.

a Peak magnitude converted from Prieto et al. (2007).

b Peak magnitude estimated from Holtzman et al. (2008) light curve.

c Peak magnitude from Li et al. (2011b).

${ }^{\mathrm{d}}$ Peak magnitude estimate based on description in Dilday et al. (2008). Remaining magnitude estimates from Kessler et al. (2009). Missing $M_{R}$ values result from events discovered at the beginning or end of the search period for which maximum light is not well constrained, or because photometry is not available (9739 and 11092). Also note that the values in the $M_{R}$ column are pseudoabsolute magnitudes that have not been corrected for host galaxy extinction.

magnitudes to the well-constrained sample. Photometry is not available for the other two photometrically selected events (SDSS-II SN09739 and SN11092), so we cannot include these. The peak $R$-band pseudo-absolute magnitudes (corrected to $H_{0}=71 \mathrm{~km} \mathrm{~s}^{-1} \mathrm{Mpc}^{-1}$ ) for the volume-limited SDSS-II sample are listed in Table 2 .

\subsection{Comparison of the LOSS and SDSS-II Pseudo-absolute Magnitude Distributions}

A comparison of the LOSS SNe Ia luminosity function and the SDSS-II first year volume-limited sample shows a striking result: The samples do not agree (Figure 4). About $66 \%$ of the LOSS SNe Ia are fainter than -19 mag, but only about $27 \%$ of the events in the SDSS-II sample are this faint. We discuss the implications of this disagreement in Section 8. Only SN 2005gj, which appears to draw its power through interaction with a hydrogen shell and could technically be classified as a Type IIn, approaches the luminosity of our higher luminosity events, such as SN 2007if (Scalzo et al. 2010; Yuan et al. 2010). 


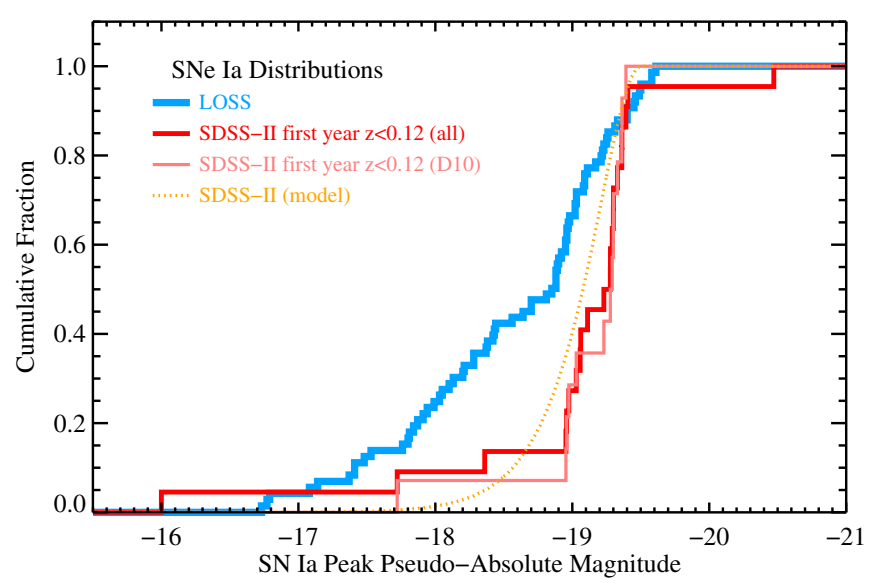

Figure 4. Absolute $\mathrm{SNe}$ Ia magnitude distributions from the LOSS (blue) and SDSS-II (red) volume-limited samples. The pink line is the subset from the volume-limited SDSS-II sample that was used in the rate calculation of Dilday et al. (2010). The dotted orange line is the model pAMD assumed in the SDSS-II rate calculation; we use this model to determine an SNe Ia rate that can be directly compared to the SDSS-II value. For the pseudo-absolute magnitudes, the observed magnitudes are corrected for Galactic extinction and the distance moduli are removed, but the host extinction is left uncorrected.

(A color version of this figure is available in the online journal.)

Also shown in Figure 4 is the $R$-band pAMD model that was assumed in calculating the SDSS-II SNe Ia rates (Dilday et al. 2010), which also appears to differ from the selected SDSS-II population. From a K-S test, we find that there is only about a $4 \%$ chance that the volume-limited SDSS-II SNe Ia sample is consistent with the attenuated, bimodal-Gaussian model of Dilday et al. (2010). The reddening distribution assumed in the model predicts that it is extremely improbable to have highly extincted events such as SDSS-II SN09266 (again, this was not spectroscopically confirmed and not included in Dilday et al. 2010). We repeated the K-S test using only the members of the volume-limited sample that were also used by Dilday et al. (2010) in their rate calculation and find the probability that these events were drawn from the assumed model is only about $3 \%$. Except for a few low-luminosity events, the observed SDSS-II sample is systematically biased to higher luminosities than the model predicts. We discuss the SDSS-II model as applied to the ROTSE-IIIb sample in Section 7.

\section{DETECTION EFFICIENCY}

In this section, we determine the detectability of sources as a function of observed magnitude relative to the limiting magnitude of a given image. This curve will be used later to determine the probability of detecting simulated sources with various observed magnitudes, which is needed to calculate our overall survey efficiency.

To determine the probability that a source of a given brightness will be detected, we perform a Monte Carlo simulation. We add simulated point sources to our co-added images and determine the fractions recovered as a function of magnitude. This is done relative to the limiting magnitude of the images and co-adds recorded in the logs, which is calculated from the 90th percentile magnitude of all objects extracted by SExtractor (Bertin \& Arnouts 1996). Our reference images are typically constructed from dozens of the best individual frames from each field, so they contribute negligibly to the noise on the subtracted frames. We therefore choose to calculate the de- tectability of sources directly from blank sky regions of the co-added images without performing the computationally expensive process of image subtraction. A consequence of this is that we do not account for the effects of contaminating host galaxy light. Other surveys that employ image subtraction have previously tested the effects of host light on detectability and found very little change from bright hosts to blank sky (Neill et al. 2006). Nonetheless, our procedure may overestimate our detection probability for supernovae hosted by bright galaxies (i.e., the rate may be underestimated). Our results do, however, have direct applications for objects with faint hosts or otherwise dark backgrounds.

On a given image, the sensitivity to objects of a given magnitude will vary with location in the field due to variations in the instrumental point spread function (PSF) as well as structure in the atmospheric transmissivity (e.g., passing clouds), which can be resolved by our wide field. To account for these effects, we locate a set of isolated field standards on each image to use in calculating the local PSF and zero point. We divide each image into a grid with as many cells as can be made while maintaining around 100 or more field standards in each (typically $3 \times 3$ cells), and then calculate the average zero point and PSF for each of these cells. The PSF is found using the DAOPHOT routines in IDL, and as noted above (Section 3.1), we use the USNO-B1.0 $\mathrm{R} 2$ system for our zero points.

To locate appropriate blank sky regions at which we may add our simulated point sources, we first run SExtractor on each unadulterated image with a low detection threshold to identify all objects and artifacts in the field. We then convolve the object mask image from SExtractor with the average PSF and select from the remaining, unmasked pixels when placing simulated sources. We find that even the small perturbations induced by adding a very faint $\left(m>m_{\text {lim }}+2\right)$ test source can sometimes elevate a faint $\left(m \sim m_{\text {lim }}\right)$ source previously ignored by SExtractor into a spurious detection. It is therefore important to avoid locating test sources near such areas, which is why we use a lower extraction threshold when creating the object mask.

We use the local PSF and zero point determined above to determine the shape of the test source and scale it to a randomly chosen magnitude. Using the dao_value routine, we create a thumbnail image of the source. We then add this source to the image one pixel at a time by drawing from a Poissonian distribution with the expectation values set by the thumbnail pixel values and accounting for the detector gain. We finally run SExtractor with the same parameter file used in the search. In order to simulate a statistically meaningful number of test objects without biasing the extraction process, we place only 20 test sources in each cell and repeat the simulation multiple times for each image. The minimum pitch for the test objects is set to be at least four times the average FWHM, so they do not interfere with each other. For the survey, candidates must be detected at a signal to noise greater than a set limit and they must have an FWHM between half and double the image average to be considered. Both of these quantities are effected by the noise in the image and Poisson statistics. We therefore define our detection efficiency from the number of simulated point sources that are detected by SExtractor with parameters that pass these selection cuts relative to the total number simulated. We performed this test on over 7000 co-added images made from two or more frames. For comparison, our survey includes about 55,000 master subtractions.

Figure 5 shows the average $5 \sigma$ detection efficiency curves for isolated objects. As can be seen, the shape of the curve is 


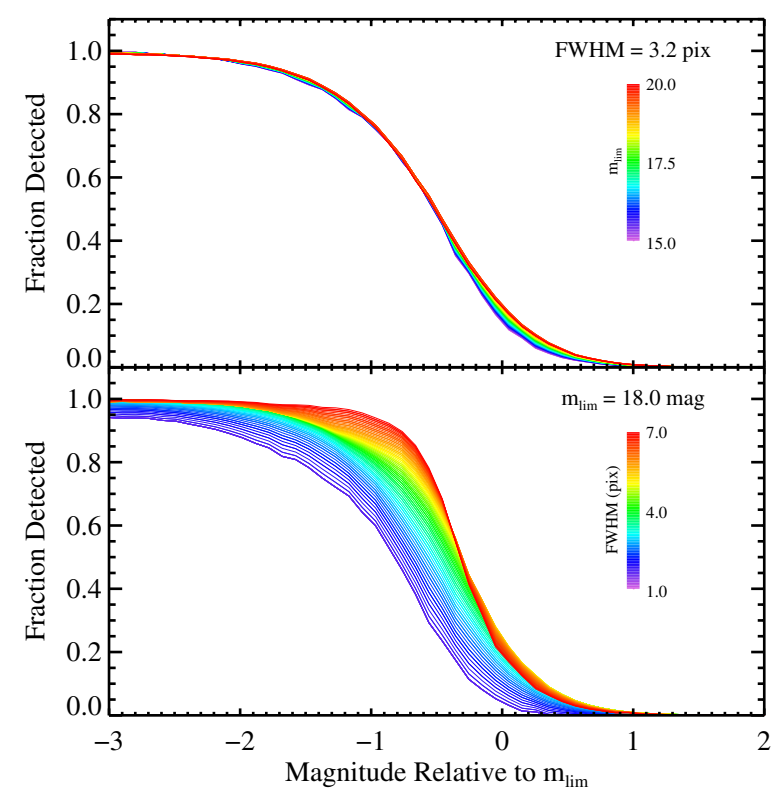

Figure 5. Detection efficiency for simulated point sources as a function of magnitude relative to the logged limiting magnitude, $m_{\mathrm{lim}}$. The top panel shows that the shape of the detection efficiency curve is nearly independent of the limiting magnitude for a fixed FWHM. The lower panel shows that for a fixed $m_{\text {lim }}$, the shape of the detection efficiency curve depends strongly on the average FWHM of the image. For both plots, the $50 \%$ completeness is brighter than the logged limit mainly because the simulations require a $5 \sigma$ detection, while the logged limits are derived from all extracted sources (i.e., $3 \sigma$ detections are included).

(A color version of this figure is available in the online journal.)

robust to changes in the limiting magnitude, but the effect from variations in the FWHM is quite noticeable.

Accurate limiting magnitudes were not recorded for the coadded images during the first half of our survey, but we do have the limits for the individual images. Using our later data, we determine an empirical relation between the limits of the individual images and the co-adds derived from them. We started by assuming that the limiting magnitudes for each individual image are approximately equal to three times the sky noise in the aperture. Inverting this, we can find the sky noise expected on the co-added frame from the sum of the individual images, and turn this into a limiting magnitude guess. Comparing these guesses to the actual values recorded in the logs over the second half of our survey, we find that the agreement is good for the deepest data, but there is a systematic offset for more shallow co-adds. We perform a linear fit to these data and use it to derive magnitude limits for the co-adds created in the beginning of the survey.

\section{SURVEY EFFICIENCY}

It is not enough to merely detect a source to count it as a discovery; each such candidate must pass additional screening to be included in our sample. This includes both machine cuts designed to reject false positives and cuts made by the humans who have the final say in elevating candidates to discoveries. Often it is straightforward to determine the effects of the machine cuts through a Monte Carlo process: Simulated supernovae drawn from the expected population are "observed" using the actual survey cadence and limiting magnitudes, and the resulting "candidates" are passed through the same machine vetting process as the real data. The sensitivity of the survey to sources with various apparent brightnesses and light curves can then be directly determined by noting the fraction of the simulated supernova that pass. The effects humans play in the ultimate selection of candidates could in principle be determined through a similar simulation if the test were performed concurrently with the actual survey to account for learned behavior. To get statistically meaningful results, the human scanner would need to be presented with an enormous volume of simulated data, which is far too large of a burden on the researchers to be done in practice, although previous studies have performed limited versions of such human experimentation, which guide our tests.

As stated above, the basic machine cuts applied to the raw candidates are (1) the FWHM should be between 0.5 and 2 times the image average, and (2) the signal to noise should be at least $2.5 \sigma$ on SUB1 and SUB2, and $5 \sigma$ for SUB. To help reduce the number of false positives created by imperfect subtraction of galaxy light, we also required that candidates near the cores of cataloged galaxies show an increase of at least $5 \%$ in flux over the reference template (given the bright limits of our survey and prior coverage of our search fields by galaxy surveys, it is fair to assume that almost all galaxies as bright or brighter than our SNe Ia sample were cataloged). We are mainly sensitive to SNe Ia brighter than -19.0 absolute, so hosts fainter than about -20.3 are unaffected by this cut. Of course, when the host galaxy is resolved, only the fraction of light coincident with the candidate matters in this percentage increase cut. For example, we recorded a $50 \%$ increase for SN $2005 \mathrm{bg}\left(M_{\text {peak }}=-19\right)$, which was discovered near the core of an $M_{r} \sim-21$ host. Candidates coincident with sources not listed in the galaxy catalogs (typically foreground stars) were required to show a $15 \%$ increase. The remaining candidates located more toward the outskirts of cataloged galaxies or in isolated regions were not required to show a minimum increase in flux. We do not include this selection criteria in our Monte Carlo simulations, so our efficiency in discovering supernovae in luminous host galaxies may be overestimated, which again means that our rate in giants may be underestimated.

The basic setup for our efficiency calculation is similar to that used in previous rate studies (cf. Dilday et al. 2008). We determine the efficiency as a function of luminosity distance by considering a series of thin shells over which the efficiency (and rate) can be assumed to be constant. In each of these shells we simulate a number of SNe Ia randomly drawn from the pAMDs discussed in Section 3, choose an appropriate light curve template and scale it to the given distance, and select a random date for maximum light. By comparing the expected magnitudes on the dates each simulated supernova would have been observed by our survey and using the actual survey detection limits on those nights, we determine the fraction of simulated SNe Ia that could have been detected in each distance bin. We take into account the detection efficiency curve discussed in Section 4 appropriate to a given observation based on the limiting magnitude and FWHM recorded (or estimated). For each observation epoch of each simulated supernova, we draw random numbers and compare these to the detection probabilities to determine whether a simulated detection is made on each of the SUB1, SUB2, and SUB. For each of our 177 fields, we simulated $100 \mathrm{SNe}$ Ia in each of 360 distance shells spaced logarithmically from 40 to $1000 \mathrm{Mpc}$, which totals to over six million simulations for each pAMD assumed. To naturally account for the changing number of survey fields, we allow simulated supernovae to peak anytime in any field during the entire survey period including off season. 

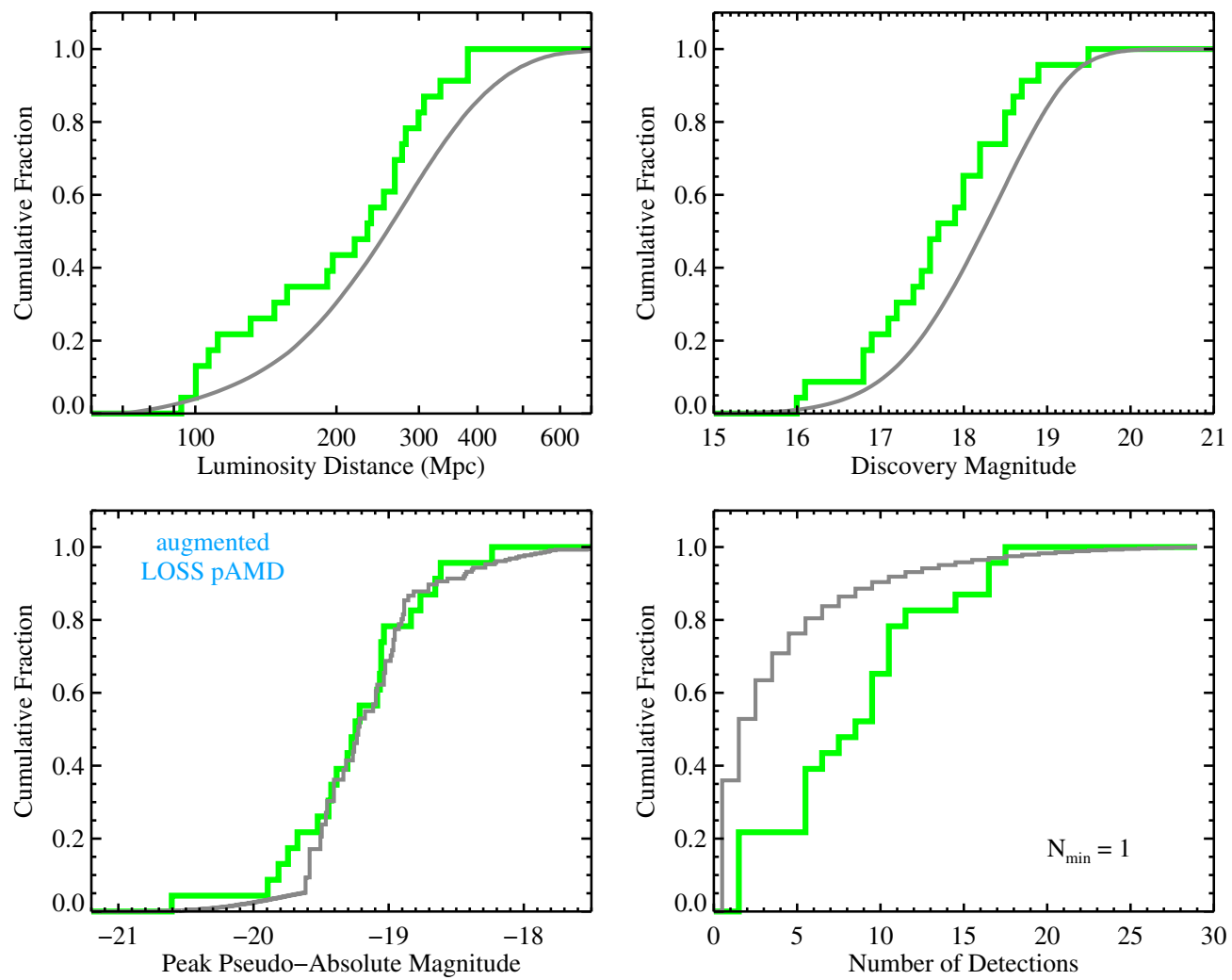

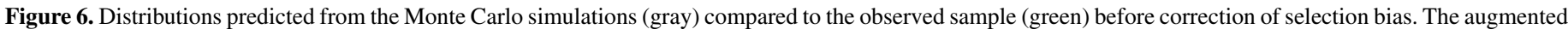
LOSS pseudo-absolute magnitude distribution (see Section 3.2) is assumed ( $\mathrm{Li}$ et al. 2011b).

(A color version of this figure is available in the online journal.)

To assess how accurately these simulations model the actual survey, we compare the luminosity distance, discovery magnitude, peak absolute magnitude, and detection number distributions predicted by the Monte Carlo simulations to the observed distributions. Figure 6 shows that the survey model discussed thus far is a poor representation of the true sample if the augmented LOSS pAMD is adopted (the same result holds if the SDSS-II model pAMD is used). The model predicts that the SNe Ia should be further away than observed and discovered at magnitudes fainter than observed, and the simulated supernovae are typically detected on fewer nights. This last result is key to understanding the failure of the model thus far. The model predicts that $>35 \%$ of the SNe Ia should be detected by the automatic search selection cuts on exactly one night. After all, the largest volume element is for the most distant events that are only detectable at their peak. Since we have zero SNe Ia detected on just one night, it would seem that our survey is biased against such "one-nighters." A possible explanation for this is the influence of the human scanners who have the final say in selecting targets. Although it was never a formal requirement of the search that events be detected on more than one night (and in fact, we triggered spectroscopic follow-up more than once on the same night based on a first detection), the informal requirement that candidates "look good" may have created a bias against the weakest detections. Indeed, Dilday et al. (2008) studied the response of human scanners to simulated supernovae and determined that there is such a bias against one-nighters.

To account for our apparent selection bias against weak detections, we apply a cut to the simulations and the real sample alike on the number of nights detected. We find that setting a minimum number of five nights, the model and actual distributions agree for both the augmented LOSS pAMD and the SDSS-II model. This cut preferentially removes the most distant supernovae with the faintest observed magnitudes, so after applying this single correction, all of the model distributions considered agree with the actual observed sample (Figures 7 and 8). The minimum number of detections was set by calculating the probability that the observed sample was drawn from the model distribution via a K-S test. We assign a consistency probability, $P_{\mathrm{KS}}$, by randomly drawing from the model distribution and comparing the maximum displacement in the cumulative distributions of the model and each draw. For four or fewer detections and the LOSS pAMD, only $0.3 \%$ (or $0.4 \%$ with the SDSS-II pAMD model) or less of the random draws have a displacement as large or larger than the real data compared to the models, so we reject these models. With a minimum of five detections, the probability rises to $9 \%(5 \%)$, so we cannot reject the null hypothesis that the data were drawn from this model distribution. In this case, the probabilities for the other three distributions are considerably higher, so the model does not appear to be inconsistent with the data. Our final, distance-dependent search efficiency is plotted in Figure 9.

We also tested the effects of systematically shifting the input pAMDs in brightness. If we shift the augmented LOSS pAMD 0.3 mag fainter, both the predicted distance and absolute magnitude distributions become incompatible with the observed distributions (as above we require pipeline detections on five or more nights). Shifting the LOSS pAMD 0.2 mag brighter, the predicted and observed distance distributions fall into agreement, but the absolute magnitude distributions are not compatible. With the SDSS-II pAMD model, a shift of either 0.2 mag fainter or brighter can be ruled out. This demonstrates 

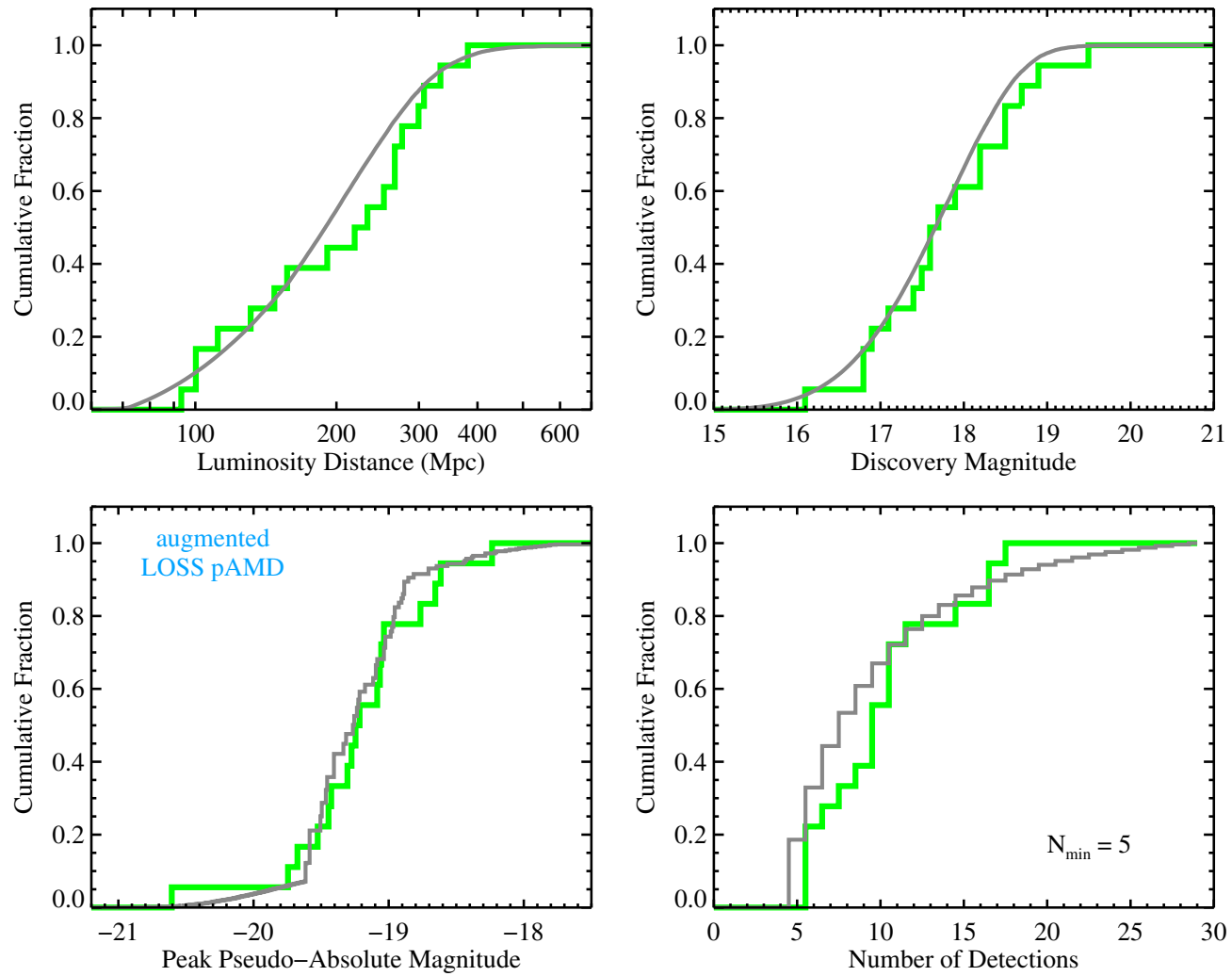

Figure 7. Distributions predicted from the Monte Carlo simulations (gray) compared to the observed sample (green) after correction for selection bias. The augmented LOSS pseudo-absolute magnitude distribution (see Section 3.2) is assumed ( $\mathrm{Li}$ et al. 2011b).

(A color version of this figure is available in the online journal.)

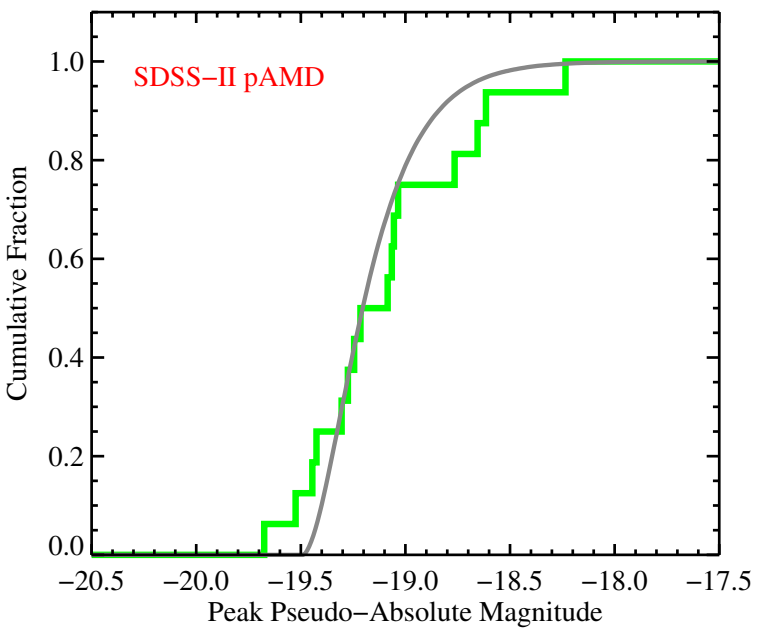

Figure 8. Peak pseudo-absolute magnitude distribution recovered from the Monte Carlo simulations assuming the SDSS-II model magnitudes (gray; see Section 3.3) compared to the observed sample (green) after correction for selection bias.

(A color version of this figure is available in the online journal.)

that we would be able to detect whether the magnitude system of the pAMD employed was systematically biased with respect to our unfiltered magnitudes by more that expected from our checks in Section 3. We will use the allowed magnitude offsets to estimate our systematic error in Section 8.

\section{HOST GALAXIES}

Li et al. (2011a) found that lower luminosity galaxies produced more SNe Ia per unit luminosity than high-luminosity

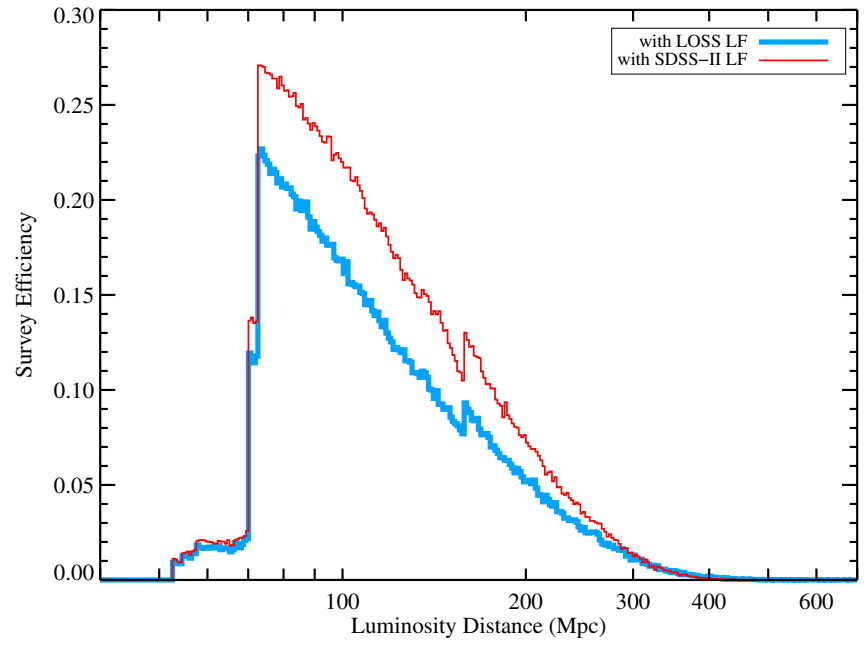

Figure 9. Search efficiency assuming the modified LOSS pseudo-absolute magnitude distribution (blue) or the SDSS-II model (red) and averaged over all fields and the full survey time span (including unsearched periods). The sudden jumps in efficiency mark the minimum allowable distances for various fields (e.g., targets in the Virgo fields must be at least $72 \mathrm{Mpc}$ away to meet our selection cuts).

(A color version of this figure is available in the online journal.)

hosts. In order to compare the host luminosity distribution of our sample to the SDSS-II and the expectations of the LOSS luminosity specific rates coupled with a galaxy luminosity function, we have measured ugriz photometry for each supernova host. We make deep stacks from the SDSS DR7 data (Abazajian et al. 2009) in each of the five bands, excluding data obtained, while the supernovae were active (assumed to be -20 days $<$ 
$t_{\max }<100$ days in the rest frame), and retain the median using a slightly modified version of the Montage package. ${ }^{11}$ Cuts on the sky background and PSF size are used to reject the worst images (about a third of the available data). We identify the host galaxies following Sullivan et al. (2006). Briefly, we use the SExtractor shape parameters $\left(C_{x x}, C_{x y}\right.$, and $\left.C_{y y}\right)$ to define an ellipse for each object in the field, and we identify the host as the object that includes the supernova position inside its ellipse with the smallest possible scaling unless the scaling factor, $R$, is greater than five. If this is the case, we perform forced photometry at the location of the supernova in a $3^{\prime \prime}$ diameter aperture. Otherwise we run SExtractor in dual image mode to measure the host galaxy photometry in consistent aperture sizes on the best-matching host. We choose to perform the object detection and aperture definition on the $r$-band images.

A few host galaxies are worthy of special mention: (1) The host of SN 2007if is not detected in the co-added SDSS data. We instead use the $g$-band magnitude of the host reported by Childress et al. (2011) to scale a spectrum of the host galaxy and then measure synthetic photometry in the SDSS bands from this. (2) The LOSS team assigned the giant galaxy KUG $1259+286$ as the host of SN 2005ck, but the SDSS redshift for this galaxy is incompatible with the supernova (Pugh et al. 2005; Leaman et al. 2011). There is a small galaxy nearby, SDSS J130218.65+282046.8, for which we find a spectroscopic redshift consistent with SN 2005ck; however, this galaxy is slightly too far away to meet the host selection criteria described above (as would be KUG 1259+286), and it is therefore considered hostless. Identified or not, the host must be a dwarf, so this should not affect our conclusions. (3) SN 2007sp is located a considerable distance $(R>10)$ from a large galaxy at a similar SDSS-derived redshift. Although this may yet be the host, for consistent application of the Sullivan et al. (2006) selection method, we must consider this target to be hostless. (4) There is a galaxy coincident with the location of SN 2007kh, but spectroscopic follow-up shows this to be a $z \sim 0.5$ galaxy, which is well in the background. The true host of SN 2007kh is therefore a mystery, so we simply adopt the photometry of the background source, assume the redshift of SN 2007kh, and take this as an upper limit on the host luminosity. (5) The location of SN 2007op is outside of the SDSS DR7 footprint; however, the host is included in DR8, so we adopt the photometry from the DR8 pipeline (Aihara et al. 2011).

In addition, we perform the same analysis on the full set of 516 spectroscopically confirmed, probable, or photometrically probable $z<0.3$ SNe Ia from the SDSS-II (Dilday et al. 2010). The larger number of visits to Stripe 82 over the course of the SDSS-II results in co-added images (again excluding those contaminated by supernova light) with limiting magnitudes considerably deeper than the search itself. As a result, even $M \sim-17$ dwarf galaxies can be detected out to the $z \sim 0.3$ limit of the sample. As with the ROTSE-IIIb sample, we manually reviewed the selection of each host galaxy and noted any special circumstances that might produce misleading results (the potential error rate appears similar for the ROTSE-IIIb and SDSS-II samples). As needed, we adjusted the SExtractor parameter DEBLEND_MINCONT either to ensure that large galaxies were not inadvertently split into smaller pieces or to attempt to split a likely galaxy blend. The latter issue was not always resolved, both for the ROTSE-IIIb and SDSS-II hosts. Of particular note, a limited number of SDSS-II hosts appear to be dwarf galaxies

11 http://montage.ipac.caltech.edu/ heavily blended with (if not merging into) larger galaxies, such as SDSS-II SN03592. When such blends are not separated by SExtractor, we perform forced aperture photometry at the location of these supernovae to better reflect the luminosity of the dwarf hosts.

Since the host galaxies are distributed over a range of redshifts, we must perform $K$-corrections to compare the galaxy luminosities in the same rest-frame bandpasses. This is accomplished using the kcorrect . pro package, version 4.2 (Blanton $\&$ Roweis 2007). For comparison against the literature, we calculate absolute $B$ - and $K$-band magnitudes for our hosts in the Vega system. These are recovered from the best-fit galaxy template through kcorrect . pro. We compared our derived $K$-band magnitudes (based only on the optical SDSS photometry) for a few low-redshift hosts to detections in the Two Micron All Sky Survey (Skrutskie et al. 2006) and found good agreement.

The four lowest luminosity hosts from the ROTSE-IIIb and nearby SDSS-II samples are shown in Figure 10 and the four highest luminosity hosts from each sample are shown in Figure 11. In Figure 12, we compare the host galaxies of the ROTSE-IIIb sample to the 20 hosts from the SDSS-II sample with redshifts $z<0.09$ (Dilday et al. 2010). These two samples cover a similar redshift span and can thus be compared directly. As can be seen, our sample includes relatively few high-luminosity host galaxies and, of particular interest, a number of very low luminosity hosts. A K-S test gives only a $4 \%$ chance that these ROTSE-IIIb and low-redshift SDSS-II samples are drawn from the same population. If we assign SDSS-II SN03592 to the larger host, this probability drops to just $1 \%$. The agreement likelihood with the full population of galaxies hosting the SNe Ia from the SDSS-II rate calculation is about $0.2 \%$. For this larger sample, a small fraction of cases that have undetected hosts also have limiting magnitudes brighter than detected ROTSE-IIIb hosts, so the exact distribution below $M_{B} \sim-17$ is uncertain. However, this does not affect our key result: $43 \%$ of the ROTSE-IIIb hosts are $M_{B}>-18$ mag dwarf galaxies, while only $17 \%$ of the full SDSS-II and 2 out of 20 from the $z<0.09$ sample are hosted by such dwarfs (including SDSS-II SN03592).

Figure 12 also shows the host galaxy distributions from the SNLS (Astier et al. 2006). As with the SDSS data, we measure the host galaxy photometry using SExtractor in dual image mode, this time using the $i$ band as reference, and we determine the rest-frame absolute magnitudes with kcorrect.pro. We use the MegaPipe median image stacks provided through CADC (Gwyn 2008). To check whether these measurements are biased by the inclusion of images with the supernovae detected in the determination of the medians, we compare our $i$-band observer frame measurements to Sullivan et al. (2010). Surprisingly, we find that our measurements are actually systematically fainter than the Sullivan et al. (2010) values by $\sim 0.3 \mathrm{mag}$. For example, for the host of SNLS 03D1ar, we find $m_{i}=19.84$ without correcting for 0.05 mag of Galactic reddening (Schlegel et al. 1998). This agrees with the value in the MegaPipe catalog, and it is consistent with the SDSS DR8 $(19.88 \pm 0.04)$, but it is fainter than reported in Sullivan et al. (2010), who found $m_{i}=19.57$. Aside from this systematic offset, we do not find any evidence that our photometry is biased by the supernova light even in the faintest hosts. It is interesting that the $B$-band distributions of the SDSS-II and SNLS host galaxies appear to diverge toward the bright end, but there is a $12 \%$ chance that a random draw from the SDSS-II population would lead to an equal or larger displacement. As with 

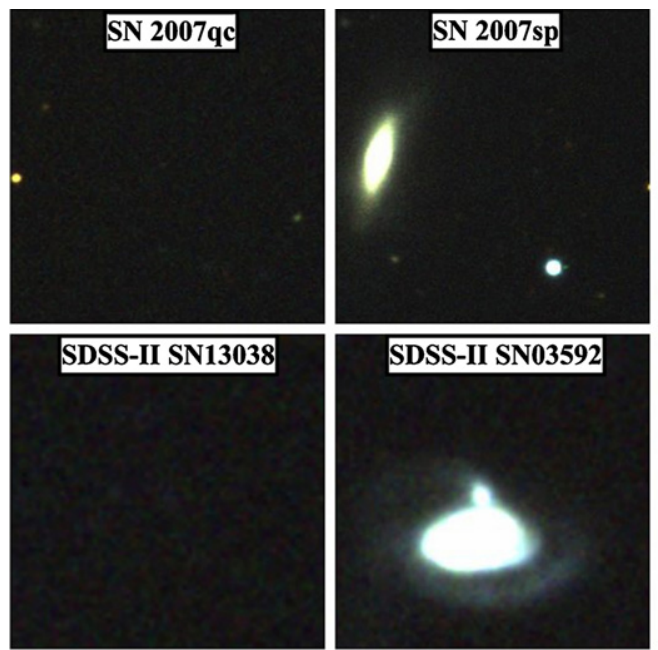
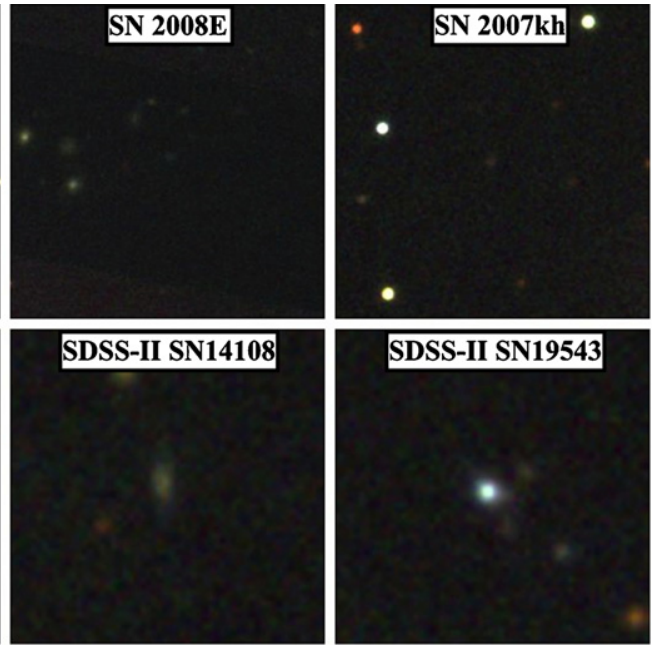

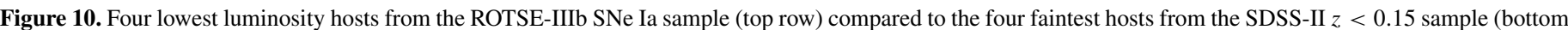

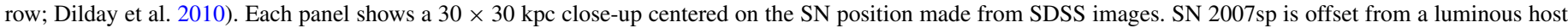

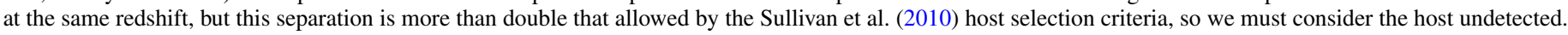

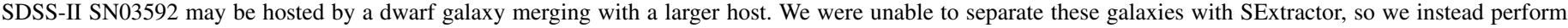

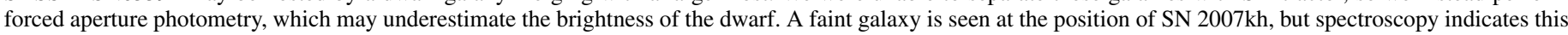
is a background source, so we take its measured brightness as an upper limit on the actual host.

(A color version of this figure is available in the online journal.)
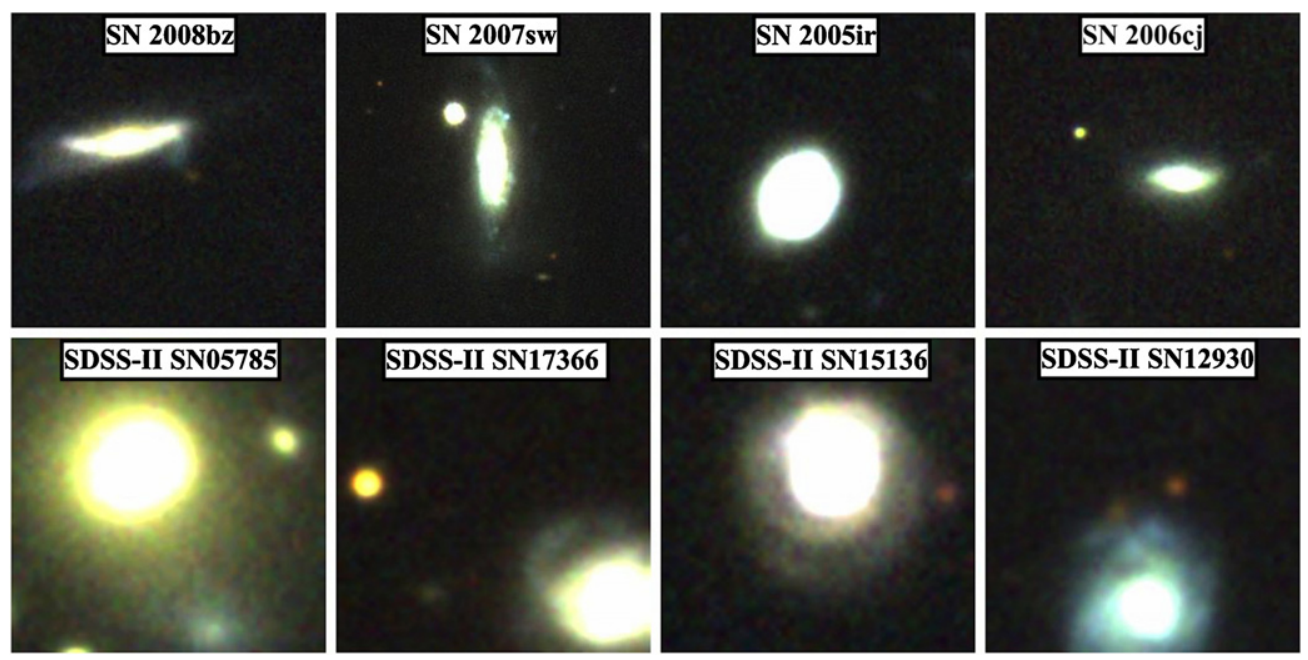

Figure 11. Same as Figure 10, but for the four highest luminosity hosts in the ROTSE-IIIb sample (top row) and the SDSS-II (bottom row).

(A color version of this figure is available in the online journal.)

the SDSS-II, the deep co-adds are sufficiently deep to detect all SNLS hosts brighter than about $M_{B} \sim-17$, and again the ROTSE-IIIb SNe Ia prefer fainter hosts.

Although LOSS targets specific galaxies, which biases the sample against low-luminosity hosts, they adopt the Kochanek et al. (2001) $K$-band galaxy luminosity function and factor in their rate-size relation to determine the volumetric $\mathrm{SNe}$ Ia rate (Li et al. 2011a). We include the $K$-band host galaxy distribution expected by the LOSS rate calculation in the lower panel of Figure 12. Clearly, this expected distribution is different than the observed SDSS-II and SNLS distributions. Specifically, LOSS assumes that $80 \%$ of SNe Ia in a given volume should come from hosts fainter than about $K>-23.4 \mathrm{mag}$, but the SDSS-II finds only about $40 \%$ of its $z<0.09$ hosts are fainter than this luminosity. This result holds qualitatively even if the correction for the rate-size relation is removed; among the giants, the
SDSS-II sample shows a preference for the higher luminosity hosts. Moving to lower luminosities, the ROTSE-IIIb sample shows a considerably higher fraction in dwarf galaxies.

As explained in Section 5, our selection process for ROTSE-IIIb may have a slight bias against high-luminosity hosts. In the next section, we calculate the volumetric rates of SNe Ia in dwarfs and giants for comparison against other samples. For convenience, we will label galaxies fainter than $M_{B}>-18$ as "dwarfs," and more luminous galaxies as "giants." When discussing the $K$-band magnitudes of the hosts, we will consider $M_{K}>-20$ galaxies to be dwarfs, since the $B-K$ colors for many of our $M_{B} \sim-18$ hosts are around 2 mag. If our sample's apparent preference of low-luminosity hosts is a result of a selection effect, then our SNe Ia rate in giants should be lower than the actual rate, while the rate in dwarfs should be accurate. We compare our values to published rates as a check. 


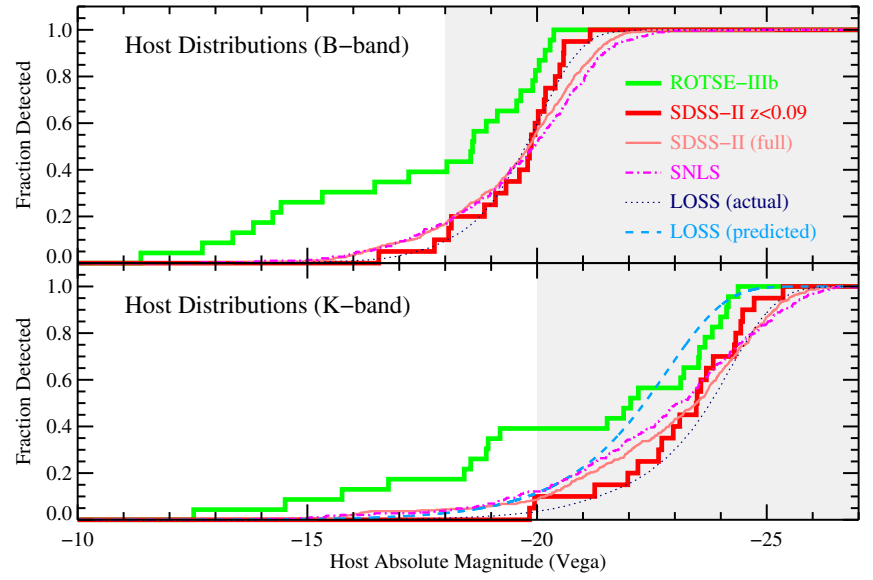

Figure 12. Absolute magnitude distributions for the host galaxies of SNe Ia. A few of the hosts fainter than $M_{B} \gtrsim-17$ were not detected. We employ upper limits for these events, but this does not affect our results. The host galaxies of the ROTSE-IIIb supernovae (green line) are typically fainter than hosts from the SDSS-II search in a similar redshift range (red line), and the ROTSE-IIIb sample includes a larger fraction of dwarf hosts than seen or predicted through the other surveys shown.

(A color version of this figure is available in the online journal.)

\section{RATES}

In this section, we calculate the volumetric $\mathrm{SNe}$ Ia rate, $\mathcal{R}$, using

$$
\mathcal{R}=\frac{N_{\mathrm{obs}}}{\epsilon V t},
$$

where $N_{\text {obs }}$ is the observed number of events, $t$ is the proper time of the survey, $V$ is the comoving volume surveyed, and $\epsilon$ is the efficiency factor that gives the estimated fraction of events actually discovered to the true number of events in the time and volume covered (i.e., $\epsilon \leqslant 1$ ).

We have 18 SNe Ia appropriate to the augmented LOSS pAMD and 16 appropriate for the SDSS-II model that were detected on five or more nights (see Sections 3 and 5). We calculated the denominator of Equation (7) in Section 5 as a sum over a series of logarithmically spaced distance bins to account for the decline in survey efficiency with distance (see Figure 9). The proper time for the survey is calculated by correcting the observer frame span for time dilation in each luminosity distance bin. The survey period was set from 2004 November 1 through 2009 January 31, with the former date reflecting the month the survey began and the latter set to the end of the standard 2008B astronomical semester. The final term for the survey volume is calculated for each distance bin by integrating the comoving volume element of the Friedmann-Robertson-Walker metric with our chosen cosmological parameters (a flat, $H_{0}=71 \mathrm{~km} \mathrm{~s}^{-1} \mathrm{Mpc}^{-1}$ and $\Omega_{m}=0.27$ universe) and factoring in the fraction of sky covered by our survey.

Special care must be taken to determine the actual survey area since the galaxy clusters of the survey are covered by an irregular grid of overlapping fields (see Figures 1 and 2). The typical pointing error of ROTSE-IIIb is about $\pm 0.05 \mathrm{deg}$, so we assume the reference templates, which represent the intersection of numerous individual images, cover $1.75 \times 1.75 \mathrm{deg}$ each. The actual pitch for the main search fields is $1.65 \mathrm{deg}$, so this is the effective size for fields bordered on all sides. To determine the total survey area, we construct an all sky register similar to the images in Figures 1 and 2, and then total all of the sky

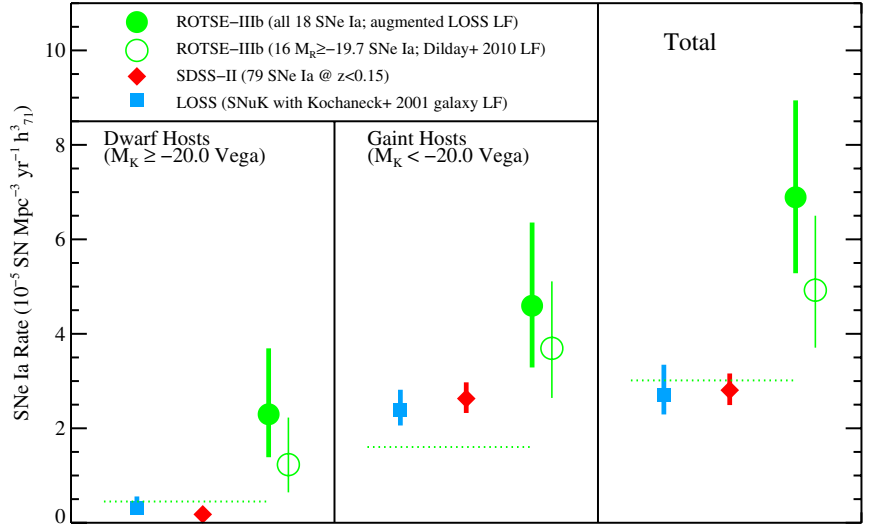

Figure 13. Volumetric SNe Ia rates. The right panel shows the total volumetric rate from ROTSE-IIIb (green circles; this work), SDSS-II (red diamond; Dilday et al. 2010), and the LOSS SNuK rate combined with a $K$-band galaxy luminosity function (blue square; Li et al. 2011a; Kochanek et al. 2001). The vertical bars show the statistical error only. The panels to the left show the contributions to the total volumetric rate from dwarf and giant hosts. The dotted horizontal lines mark the $3 \sigma$ lower limits for the complete ROTSE-IIIb samples in each group. The filled green circle shows the ROTSE-IIIb rates computed assuming the LOSS SNe Ia pseudo-absolute magnitude distribution (Li et al. 2011b), and the open circles show the values derived using the same SNe Ia distribution assumed for the SDSS-II calculation (Dilday et al. 2008, 2010) and removing spectroscopically confirmed SNe Ia in a similar manner to the SDSS-II vetting process.

(A color version of this figure is available in the online journal.)

area visited at least once during the survey period. Our total sky coverage is $499.0 \mathrm{deg}^{2}$.

Adopting the LOSS SNe Ia pAMD with a supplemental population of high-luminosity events (assumed to be $1 \%$ of the total population; see Section 3.2) we find a rate of $\left(6.9_{-1.6}^{+2.1}\right) \times$ $10^{-5} \mathrm{SNe}$ Ia $\mathrm{Mpc}^{-3} \mathrm{yr}^{-1} h_{71}^{3}$ (statistical error only). With the SDSS-II model pAMD, the volumetric rate drops to the lower (but statistically consistent) value of $\left(4.9_{-1.2}^{+1.6}\right) \times 10^{-5}$ $\mathrm{SNe}$ Ia $\mathrm{Mpc}^{-3} \mathrm{yr}^{-1} h_{71}^{3}$. These total rates and the rates specific to dwarf and giant hosts are shown in Figure 13 and listed in Table 3.

For comparison, the SDSS-II found an SNe Ia rate of $\left(2.81_{-0.32}^{+0.35}\right) \times 10^{-5} \mathrm{SNe} \mathrm{Ia} \mathrm{Mpc}{ }^{-3} \mathrm{yr}^{-1} h_{71}^{3}$ using their $z<0.15$ SNe Ia and assuming the rate to be constant out to this limit (Dilday et al. 2010). Since the SNe Ia rate increases with redshift and even the lowest SDSS-II redshift bin extends out to larger distances than the ROTSE-IIIb sample, the SDSS-II rate may be slightly inflated with respect to our more nearby rate measurement. In any case, the SDSS-II rate is below our $3 \sigma$ lower limit derived with the augmented LOSS pAMD and may be incompatible with the SDSS-II model pAMD as well at about the $2 \sigma$ level. We split the samples into dwarf and giant hosts and find that while the ROTSE-IIIb rates in giants are marginally consistent with the SDSS-II results, our rate in dwarfs is significantly higher than the SDSS-II with either the augmented LOSS or SDSS-II model pAMD.

To convert the LOSS host luminosity specific SNe Ia rate into a volumetric rate, we follow similar steps as reported in $\mathrm{Li}$ et al. (2011a), but with an important difference. We start with the rate-size relation and SNuK measurement from LOSS and combine this with the local galaxy $K$-band luminosity function (Kochanek et al. 2001). Like the LOSS estimate, the calculation is done separately for early- and late-type galaxies, and then combined to give the final result. The published LOSS rate is determined by multiplying the local galaxy $K$-band luminosity 
Table 3

Type Ia Supernova Rates

\begin{tabular}{lcccccc}
\hline \hline Survey & $N_{\text {Ia,dwarfs }}$ & Dwarfs & $N_{\text {Ia, giants }}$ & Giants & $N_{\text {Ia,tal }}$ & Total \\
\hline LOSS & $\ldots$ & $0.31_{-0.10}^{+0.25}$ & $\ldots$ & $2.39_{-0.33}^{+0.43}$ & $\ldots$ & $2.70_{-0.41}^{+0.65}$ \\
SDSS-II $(z<0.15)$ & 5 & $0.18_{-0.08}^{+0.12}$ & 74 & $2.63_{-0.30}^{+0.34}$ & 79 & $2.81_{-0.35}^{+0.35}$ \\
ROTSE-IIIb (w/LOSS LF) & 6 & $2.31_{-0.92}^{+1.41}$ & 12 & $4.63_{-1.32}^{+1.78}$ & 18 & $6.94_{-1.62}^{+2.07}$ \\
ROTSE-IIIb (w/SDSS-II LF) & 4 & $1.23_{-0.59}^{+1.00}$ & 12 & $3.69_{-1.05}^{+1.42}$ & 16 & $4.92_{-1.22}^{+1.58}$ \\
\hline
\end{tabular}

Note. Rates in units of $10^{-5} \mathrm{SNe} \mathrm{Ia} \mathrm{Mpc}^{-3} \mathrm{yr}^{-1} h_{71}^{3}$.

densities $\left(j_{\text {early }}\right.$ and $\left.j_{\text {late }}\right)$ by average values of their $\mathrm{SNuK}$, which are determined from a weighted average involving the number densities from the galaxy luminosity functions. We choose to recalculate the LOSS rate through what we believe to be a more direct approach. We simply multiply the galaxy luminosity function by the rate-size relation function and integrate. With our approach, we find the volumetric rate implied by the LOSS sample is $\left(2.70_{-0.41}^{+0.65}\right) \times 10^{-5} \mathrm{SNe} \mathrm{Ia} \mathrm{Mpc}^{-3} \mathrm{yr}^{-1} h_{71}^{3}$. This is consistent with, if not slightly lower than, the published LOSS rate (Li et al. 2011a). We compute the statistical error through a Monte Carlo calculation including the uncertainty in the galaxy luminosity function, which gives a somewhat larger confidence interval than reported by LOSS. This rate is consistent with the SDSS-II rate, which is an odd result given the apparently different SNe Ia populations studied (see Section 3.4). Thus, as was the case with the SDSS-II rate, the total rates we derive from our ROTSE-IIIb sample are higher at the $3 \sigma$ level. We also determine the LOSS rate in dwarfs and giants by simply changing the integration interval. Again, LOSS and SDSS-II derive rates that are consistent with each other for dwarfs, giants, and the total host population even though their SNe Ia populations are apparently distinct (see further discussion in Section 8).

Using either SNe Ia pAMD, our rate in giants is comparable to SDSS-II and LOSS, so there is no evidence that our selection process is biased against high-luminosity hosts. However, with the LOSS distribution, our rate in dwarf hosts is significantly higher than that of either SDSS-II or LOSS, and with the SDSSII model pAMD, our rate is higher at about the $2 \sigma$ level.

\section{CONCLUSIONS}

We have used the background population of supernovae discovered by the ROTSE-IIIb telescope over a four year period to derive the volumetric rate of SNe Ia. Our rate is somewhat sensitive to the SNe Ia pAMD assumed in deriving our overall search efficiency, and we find that recent rates studies have used different, incompatible pAMDs. We have calculated our rates using both the LOSS pAMD augmented with a small population of high-luminosity events (1\% of the total) and the same pAMD model (and similar sample cuts) employed in the SDSS-II rate measurement to quantify our distance-dependent search efficiency. With the augmented LOSS pAMD, we find a rate that is more than double published values in a similar redshift range. This rate is higher than the SDSS-II or LOSS rates at $99.9 \%$ confidence (ignoring systematics for the moment). Similarly, performing the calculation on our "normal-bright" SNe Ia in a manner directly comparable to the SDSS-II measurement (including the same pAMD model), our rate is still nearly double that found by the SDSS-II out to a slightly higher redshift. In this case, our rate is only higher than the SDSS-II value at the $2 \sigma$ confidence level, however.
The LOSS pAMD is utterly incompatible with the SDSS-II model, which, in turn, is incompatible with the actual volumelimited sample from the first year SDSS-II. Neither the SDSS-II model nor the LOSS pAMD allow for events as bright as SN 2007if, so it would seem that none of the available distributions account for the full population of SNe Ia. Additionally, the large extinction value reported for the photometrically selected SDSS-II SN09266 suggests that these distributions may also fail to account for the true distribution of host absorptions. Down to about $-18.8 \mathrm{mag}$, the LOSS and SDSS-II model pAMDs agree, but the LOSS sample shows almost double the relative contribution from fainter events. The solid agreement between the LOSS and SDSS-II rates is perplexing given that they are apparently sampling completely different SNe Ia populations.

The systematic errors in our rate measurement are dominated by the uncertainty in the SNe Ia pAMD, including how such distributions derived from (mostly) filtered photometry apply to our unfiltered data set. Our total rate estimate with the SDSS-II model pAMD is $30 \%$ lower than our rate with the augmented LOSS pAMD. Part of this is due to the exclusion of SNe 2007if and 2008ab from the SDSS-II model rate due to their high luminosities (both of these events also have dwarf hosts). The larger fraction of low-luminosity events with the LOSS pAMD makes up the additional difference. Our search is mainly sensitive to background supernovae with pseudoabsolute magnitudes brighter than about $M_{R}=-18.5 \mathrm{mag}$, so at least $42 \%$ of SNe Ia are definitely missed by our search if the LOSS distribution is assumed, but this fraction falls to just $8 \%$ if we use the SDSS-II model. We use our rate based on the SDSS-II model pAMD to constrain the lower bound on the SNe Ia rate and our augmented LOSS rate for the upper bound. Adding in a $\pm 0.2 \mathrm{mag}$ systematic offset to account for the allowed uncertainty in our unfiltered magnitude system with respected to the (filtered) pAMDs considered, our final $\mathrm{SNe}$ Ia rate (including $1 \sigma$ statistical errors) at a mean redshift of $\bar{z}=0.05$ is then between 3.7 and 13.3 in units of $10^{-5}$ $\mathrm{SNe}$ Ia $\mathrm{Mpc}^{-3} \mathrm{yr}^{-1} h_{71}^{3}$.

As our sample is spectroscopically complete, we do not include uncertainty for unclassified candidates. This can be an important concern for other, non-spectroscopically complete studies. For example, the SDSS has reported ${ }^{12} 561$ spectroscopically confirmed $\mathrm{SNe}$ Ia, but there are at least 1070 photometrically probable SNe Ia uncovered by the same survey, and presumably many more transient candidates in all including peculiar SNe Ia, which may not pass the photometric screening (Dilday et al. 2010; Sako et al. 2011).

There is also the question of whether all objects technically classified as $\mathrm{SNe}$ Ia from their spectra should really be grouped in the same physical category. For example, some have argued that some peculiar SNe Ia, such as SN 2005hk, may not be

\footnotetext{
12 http://sdssdp62.fnal.gov/sdsssn/snlist_confirmed_updated.php
} 
thermonuclear supernovae (cf. Valenti et al. 2009). One event in our sample, SN 2006ct, bears some resemblance to this subclass, although we note some differences as well in Section 2.1. Removing this one event would lower our total rates by about $6 \%$. On the other hand, some events grouped with CCSNe may actually be SNe Ia in disguise. In particular, some Type IIn may be better suited in the SNe Ia camp (Hamuy et al. 2003; Dilday et al. 2012). Over our survey period, we discovered three background Type IIn supernovae (SNe 2006db, 2006tf, and 2008am), but none of these have been linked to Type Ia explosions.

In using the augmented LOSS pAMD, we have attempted to measure the total SNe Ia rate including Hubble diagram outliers and other peculiar events. This should be directly comparable to the rate measured by LOSS who include SN 1991T-like, SN 1991bg-like, and SN 2002cx-like events in their sample, but this has not always been common practice in the literature. Many of the previous SNe Ia rate measurements have explicitly excluded events that deviate from the sub-sample of SNe Ia that are most useful as cosmological probes. For example, the recent SNe Ia rates measured by the SNLS (Perrett et al. 2012) are strictly valid only for SNe Ia with light curve widths close to the nominal value $(0.8<s<1.3$, but see also González-Gaitán et al. 2011). SNe Ia like SN 2003fg (a.k.a. SNLS-03D3bb) are not discussed. Similarly, the SDSS-II explicitly removed their peculiar SNe Ia, such as the interacting SN 2005gj, and the selection cut on the MLCS2k2 light curve fits biases their sample against high-luminosity, SN 1999aa-like events (Dilday et al. 2010; Kessler et al. 2009). Because of this, the SNLS and SDSS-II rates only reflect the frequency for a fraction of the larger SNe Ia population. As to the high-luminosity SNe Ia excluded by SNLS and SDSS-II, however, we note that if these constituted more than a few percent of the total population then they would completely dominate our flux-limited sample, which is not the case.

Although the significance of our rates being higher than the canonical value is low, it is worth discussing how this may have come about. One point of intrigue is our excess of SNe Ia in dwarf galaxies over what would be expected from the LOSS or SDSS-II studies. If our rate for these hosts is correct, then either the SNe Ia rate-size relation increases for the lowest luminosity hosts as compared to the range studied by LOSS, or the faint end of the galaxy luminosity function used to convert the LOSS luminosity specific rate to a volumetric rate may be undercounting the actual supply of dwarf galaxies.

Such correction factors are not required for the SDSS-II rate, so the higher frequency of SNe Ia in low-luminosity hosts is puzzling. In the full background sample, 7 out of 23 of the ROTSE-IIIb hosts are fainter than $M_{B} \geqslant-16 \mathrm{mag}$, as are 5 out of the 18 used for the rate calculation with the augmented LOSS pAMD. These fractions $(\sim 30 \%)$ are significantly larger than the 1 out of 79 SNe Ia hosts in the SDSS-II $z<0.15$ sample that have such low-luminosity hosts (hosts brighter than $M_{r} \sim-15$ mag can be detected through our analysis out to this redshift limit, but only SDSS-II SN13038 is fainter). If this difference is attributed to a selection bias against giant hosts in the ROTSE-IIIb sample, then we must increase our rate to account for the missing SNe Ia in giants. But to achieve the same low- to high-luminosity host ratio as the SDSS-II, our already high rates must be increased by factors of 4-5.

Another possibility to consider is that the SDSS-II may somehow be biased against low-luminosity hosts, but this is difficult to imagine. A remote possibility is that the use of host photo- $z$ 's in selecting targets for spectroscopic follow-up may boost the likelihood of classifying $\mathrm{SNe}$ Ia in giants rather than in dwarfs, which were unlikely to have been detected in the reference data available during the search. In that case, there would still need to be a second bias to reject the SNe Ia in dwarfs since photometrically probable yet non-spectroscopically confirmed SNe Ia were included in the SDSS-II rates (Dilday et al. 2010).

A possible contributor to the relative infrequency of dwarf hosts in the SDSS-II sample may be the cut on the MLCS2k2 light curve fits used in selecting photometrically probably SNe Ia (Dilday et al. 2010). This cut can remove high-luminosity SNe Ia, such as SN 1999aa, which tend to be associated with lower luminosity hosts (Kessler et al. 2009; Sullivan et al. 2006). However, only one of the five SNe Ia from our rate sample that fall in $M_{B} \geqslant-16$ mag hosts can be considered highly luminous: SN 2007if. Since the SDSS-II does contain a number of higher redshift SNe Ia whose hosts are not detected even in our deep co-adds, it does not appear that the SDSS-II had a selection bias against either "hostless" SNe Ia or SNe Ia in dwarf hosts, and it is not obvious why such hosts are so much more frequent in the ROTSE-IIIb sample.

Another question is why there is such a larger fraction of faint (pseudo-absolute magnitude $M_{R}>-18.8$ ) SNe Ia in the LOSS sample as compared to the SDSS-II? Despite being targeted, the LOSS galaxy sample spans a range of masses and colors, and the full LOSS galaxy sample has absolute $B$ - and $K$-band magnitude distributions that are consistent with the SDSS-II SNe Ia hosts in the $z<0.09$ range (see Figure 12). Applying the LOSS rate-size relation only to galaxies brighter than $M_{K}<-20$ in the Kochanek et al. (2001) galaxy luminosity function and selecting only the $48 \%$ of SNe Ia fainter than $M_{R}>-18.8$ in the LOSS pAMD, the minimum rate of low-luminosity SNe Ia implied is $0.4 \times 10^{-5} \mathrm{SNe} \mathrm{Ia} \mathrm{Mpc}^{-3} \mathrm{yr}^{-1} h_{71}^{3}$. In this case, the first year SDSS-II $z<0.12$ sample should contain at least six $\mathrm{SNe}$ Ia fainter than $-18.8 \mathrm{mag}$. Even with the peculiar SN 2005hk and the photometrically selected SDSS-II SN09266 (with its heavy host absorption of $A_{V} \sim 4 \mathrm{mag}$ ), there are still only three events in the SDSS-II $z<0.12$ first year sample at such low luminosities.

It is not clear how the SDSS-II could have missed the faint tail of the SNe Ia distribution (e.g., SN 1991bg-like events around $M_{B} \sim-17.2$ ), yet we know of only two such spectroscopically confirmed events discovered in the three years of the SDSS-II (2007jh and 2007mm; Mosher et al. 2012) and only one possible SN 2002cx-like event (SN 2007ie; Östman et al. 2011). Additionally, the Palomar Transient Factory (Rau et al. 2009; Law et al. 2009) has recently shown that there are perhaps even more varieties of peculiar, low-luminosity objects that may be spectroscopically classified as SNe Ia (e.g., Sullivan et al. 2011; Maguire et al. 2011), but these are apparently absent from the SDSS-II as well. The apparent lack of low-luminosity SNe Ia could mean that there was an unknown selection bias against such objects and that the total rate of SNe Ia is higher than found by the SDSS-II.

The tension between the LOSS and SDSS-II pAMDs poses a potentially worrisome systematic bias for SNe Ia rate studies. Dilday et al. (2008) argue that the SDSS-II was sensitive to even sub-luminous SNe Ia below $z<0.12$, so even if their model distribution were flawed, this would not have a significant impact on their rate calculation (but again, the dearth of low-luminosity SNe Ia as compared with the expectations of LOSS is puzzling at best and may suggest the SDSS-II has altogether missed 
some $\mathrm{SNe}$ Ia). However, if there is a low-luminosity population of SNe Ia that is not accounted for in the SDSS-II model distribution, this will lead to an overestimation of the search efficiency at the higher redshifts considered in Dilday et al. (2010), and thus an underestimation of the actual rate. In general, rate studies that push their samples out to the highest redshifts possible with their surveys have an even stronger Malmquist bias and are thus more sensitive to systematic errors in their adopted pAMDs. These distributions may also vary with redshift. It is therefore important to securely determine the true SNe Ia pAMD from complete, volume-limited surveys, as could potentially be done by the Palomar Transient Factory at low redshifts and at moderate redshifts with the Hyper-SuprimeCam on Subaru.

Another issue that may lead to bogus rate evolution measurements is the changing definition of the SNe Ia sub-population measured by different surveys. Low- to moderate-redshift surveys often use light curve fits to select SNe Ia (e.g., Dilday et al. 2010; Neill et al. 2006; Perrett et al. 2012), but the highest redshift studies often have far fewer epochs available due to the increased follow-up cost, and must therefore rely more heavily on color selection techniques (Graur et al. 2011). It is not clear that these different cuts capture identical sub-populations so that differences in the rates between different surveys may be taken directly as evidence for evolution in the SNe Ia rate. This question may be resolved by performing a rolling search similar to SDSS-II but sensitive to very high redshift SNe Ia, as will be possible with the Hyper-SuprimeCam Survey.

ROTSE-III has been supported by NASA Grant NNX08AV63G and NSF Grant PHY-0801007. Parts of this research were conducted by the Australian Research Council Center of Excellence for All-sky Astrophysics (CAASTRO), through project number CE110001020. The research of J.C.W. is supported in part by NSF Grant AST1109801. This material is based upon work supported in part by the National Science Foundation under grant No. 1066293 and the hospitality of the Aspen Center for Physics. This research made use of Montage, funded by the National Aeronautics and Space Administration's Earth Science Technology Office, Computation Technologies Project, under Cooperative Agreement No. NCC5-626 between NASA and the California Institute of Technology. Montage is maintained by the NASA/IPAC Infrared Science Archive.

\section{REFERENCES}

Abazajian, K. N., Adelman-McCarthy, J. K., Agüeros, M. A., et al. 2009, ApJS, 182,543

Aihara, H., Allende Prieto, C., An, D., et al. 2011, ApJS, 193, 29

Aldering, G., Antilogus, P., Bailey, S., et al. 2006, ApJ, 650, 510

Astier, P., Guy, J., Regnault, N., et al. 2006, A\&A, 447, 31

Badenes, C., \& Maoz, D. 2012, ApJ, 749, L11

Bertin, E., \& Arnouts, S. 1996, A\&AS, 117, 393

Blanton, M. R., \& Roweis, S. 2007, AJ, 133, 734

Cappellaro, E., Evans, R., \& Turatto, M. 1999, A\&A, 351, 459

Childress, M., Aldering, G., Aragon, C., et al. 2011, ApJ, 733, 3

Colesanti, C., Jacques, C., Pimentel, E., et al. 2006, IAU Circ., 8713, 1

Contreras, C., Hamuy, M., Phillips, M. M., et al. 2010, AJ, 139, 519

Dahlen, T., Strolger, L.-G., \& Riess, A. G. 2008, ApJ, 681, 462

Dilday, B., Howell, D. A., Cenko, S. B., et al. 2012, Science, 337, 942

Dilday, B., Kessler, R., Frieman, J. A., et al. 2008, ApJ, 682, 262

Dilday, B., Smith, M., Bassett, B., et al. 2010, ApJ, 713, 1026

Domínguez, I., Höflich, P., \& Straniero, O. 2001, ApJ, 557, 279

Filippenko, A. V., Richmond, M. W., Branch, D., et al. 1992, AJ, 104, 1543

Foley, R. J., Rest, A., Stritzinger, M., et al. 2010, AJ, 140, 1321

Frieman, J. A., Bassett, B., Becker, A., et al. 2008, AJ, 135, 338

Ganeshalingam, M., Li, W., Filippenko, A. V., et al. 2012, ApJ, 751, 142
Garavini, G., Folatelli, G., Goobar, A., et al. 2004, AJ, 128, 387

González-Gaitán, S., Perrett, K., Sullivan, M., et al. 2011, ApJ, 727, 107

Graur, O., Poznanski, D., Maoz, D., et al. 2011, MNRAS, 417, 916

Gwyn, S. D. J. 2008, PASP, 120, 212

Hamuy, M., Maza, J., Phillips, M. M., et al. 1993, AJ, 106, 2392

Hamuy, M., Phillips, M. M., Suntzeff, N. B., et al. 2003, Nature, 424, 651

Hicken, M., Wood-Vasey, W. M., Blondin, S., et al. 2009, ApJ, 700, 1097

Holtzman, J. A., Marriner, J., Kessler, R., et al. 2008, AJ, 136, 2306

Howell, D. A., Sullivan, M., Conley, A., \& Carlberg, R. 2007, ApJ, 667, L37

Howell, D. A., Sullivan, M., Nugent, P. E., et al. 2006, Nature, 443, 308

Hoyle, F., \& Fowler, W. A. 1960, ApJ, 132, 565

Jha, S., Riess, A. G., \& Kirshner, R. P. 2007, ApJ, 659, 122

Kessler, R., Becker, A. C., Cinabro, D., et al. 2009, ApJS, 185, 32

Kilic, M., Brown, W. R., Allende Prieto, C., et al. 2012, ApJ, 751, 141

Kistler, M. D., Stanek, K. Z., Kochanek, C. S., Prieto, J. L., \& Thompson, T. A. 2011, arXiv:1106.3115

Kobayashi, C., \& Nomoto, K. 2009, ApJ, 707, 1466

Kochanek, C. S., Pahre, M. A., Falco, E. E., et al. 2001, ApJ, 560, 566

Law, N. M., Kulkarni, S. R., Dekany, R. G., et al. 2009, PASP, 121, 1395

Leaman, J., Li, W., Chornock, R., \& Filippenko, A. V. 2011, MNRAS, 412, 1419

Li, W., Chornock, R., Leaman, J., et al. 2011a, MNRAS, 412, 1441

Li, W., Filippenko, A. V., Chornock, R., et al. 2003, PASP, 115, 453

Li, W., Leaman, J., Chornock, R., et al. 2011b, MNRAS, 412, 1473

Maguire, K., Sullivan, M., Thomas, R. C., et al. 2011, MNRAS, 418, 747

Mannucci, F., Della Valle, M., Panagia, N., et al. 2005, A\&A, 433, 807

Mannucci, F., Della Valle, M., \& Panagia, N. 2006, MNRAS, 370, 773

Maoz, D., \& Mannucci, F. 2012, PASA, 29, 447

Maoz, D., Mannucci, F., Li, W., et al. 2011, MNRAS, 412, 1508

Maund, J. R., Wheeler, J. C., Wang, L., et al. 2010, ApJ, 722, 1162

Modjaz, M., Kirshner, R., Challis, P., Calkins, M., \& Hutchins, R. 2005, IAU Circ., 8553, 2

Mosher, J., Sako, M., Corlies, L., et al. 2012, AJ, 144, 17

Neill, J. D., Sullivan, M., Balam, D., et al. 2006, AJ, 132, 1126

Nelemans, G., Napiwotzki, R., Karl, C., et al. 2005, A\&A, 440, 1087

Nugent, P. E., Sullivan, M., Cenko, S. B., et al. 2011, Nature, 480, 344

Östman, L., Nordin, J., Goobar, A., et al. 2011, A\&A, 526, A28

Perlmutter, S., Aldering, G., Goldhaber, G., et al. 1999, ApJ, 517, 565

Perlmutter, S., Deustua, S., Gabi, S., et al. 1997, in Proc. NATO Advanced

Study Institute, Vol. 486, Thermonuclear Supernovae, ed. P. Ruiz-Lapuente,

R. Canal, \& J. Isern (Dordrecht: Kluwer), 749

Perrett, K., Sullivan, M., Conley, A., et al. 2012, AJ, 144, 59

Phillips, M. M., Li, W., Frieman, J. A., et al. 2007, PASP, 119, 360

Pickles, A. J. 1998, PASP, 110, 863

Prieto, J. L., Garnavich, P. M., Phillips, M. M., et al. 2007, arXiv:0706.4088

Pugh, H., Li, W., Quimby, R., et al. 2005, IAU Circ., 8542, 1

Quimby, R., Höflich, P., \& Wheeler, J. C. 2007a, ApJ, 666, 1083

Quimby, R., Mondol, P., Odewahn, S. C., \& Rostopchin, S. 2006, CBET, 537,1

Quimby, R. M. 2006, PhD thesis, Univ. Texas at Austin

Quimby, R. M., Wheeler, J. C., Höflich, P., et al. 2007b, ApJ, 666, 1093

Rau, A., Kulkarni, S. R., Law, N. M., et al. 2009, PASP, 121, 1334

Sako, M., Bassett, B., Becker, A., et al. 2008, AJ, 135, 348

Sako, M., Bassett, B., Connolly, B., et al. 2011, ApJ, 738, 162

Scalzo, R. A., Aldering, G., Antilogus, P., et al. 2010, ApJ, 713, 1073

Schlegel, D. J., Finkbeiner, D. P., \& Davis, M. 1998, ApJ, 500, 525

Silverman, J. M., Ganeshalingam, M., Li, W., et al. 2011, MNRAS, 410, 585

Simon, J. D., Gal-Yam, A., Gnat, O., et al. 2009, ApJ, 702, 1157

Skrutskie, M. F., Cutri, R. M., Stiening, R., et al. 2006, AJ, 131, 1163

Sullivan, M., Conley, A., Howell, D. A., et al. 2010, MNRAS, 406, 782

Sullivan, M., Kasliwal, M. M., Nugent, P. E., et al. 2011, ApJ, 732, 118

Sullivan, M., Le Borgne, D., Pritchet, C. J., et al. 2006, ApJ, 648, 868

Tremonti, C. A., Heckman, T. M., Kauffmann, G., et al. 2004, ApJ, 613, 898

Trundle, C., Kotak, R., Vink, J. S., \& Meikle, W. P. S. 2008, A\&A, 483, L47

Umeda, H., Nomoto, K., Yamaoka, H., \& Wanajo, S. 1999, ApJ, 513, 861

Valenti, S., Pastorello, A., Cappellaro, E., et al. 2009, Nature, 459, 674

Webbink, R. F. 1984, ApJ, 277, 355

Whelan, J., \& Iben, I., Jr. 1973, ApJ, 186, 1007

Yuan, F., Akerlof, C., Quimby, R., et al. 2007a, BAAS, 38, 105.05

Yuan, F., \& Akerlof, C. W. 2008, ApJ, 677, 808

Yuan, F., Quimby, R., Chamarro, D., et al. 2008a, CBET, 1273, 1

Yuan, F., Quimby, R., Peters, C., \& Thorstensen, J. 2007b, CBET, 1059, 1

Yuan, F., Quimby, R. M., Wheeler, J. C., et al. 2010, ApJ, 715, 1338

Yuan, F., Sisson, M. D., Chamarro, D., et al. 2008b, CBET, 1308, 1

Yungelson, L. R., \& Livio, M. 2000, ApJ, 528, 108

Zwicky, F. 1938, ApJ, 88, 529 